BMC

Genomics

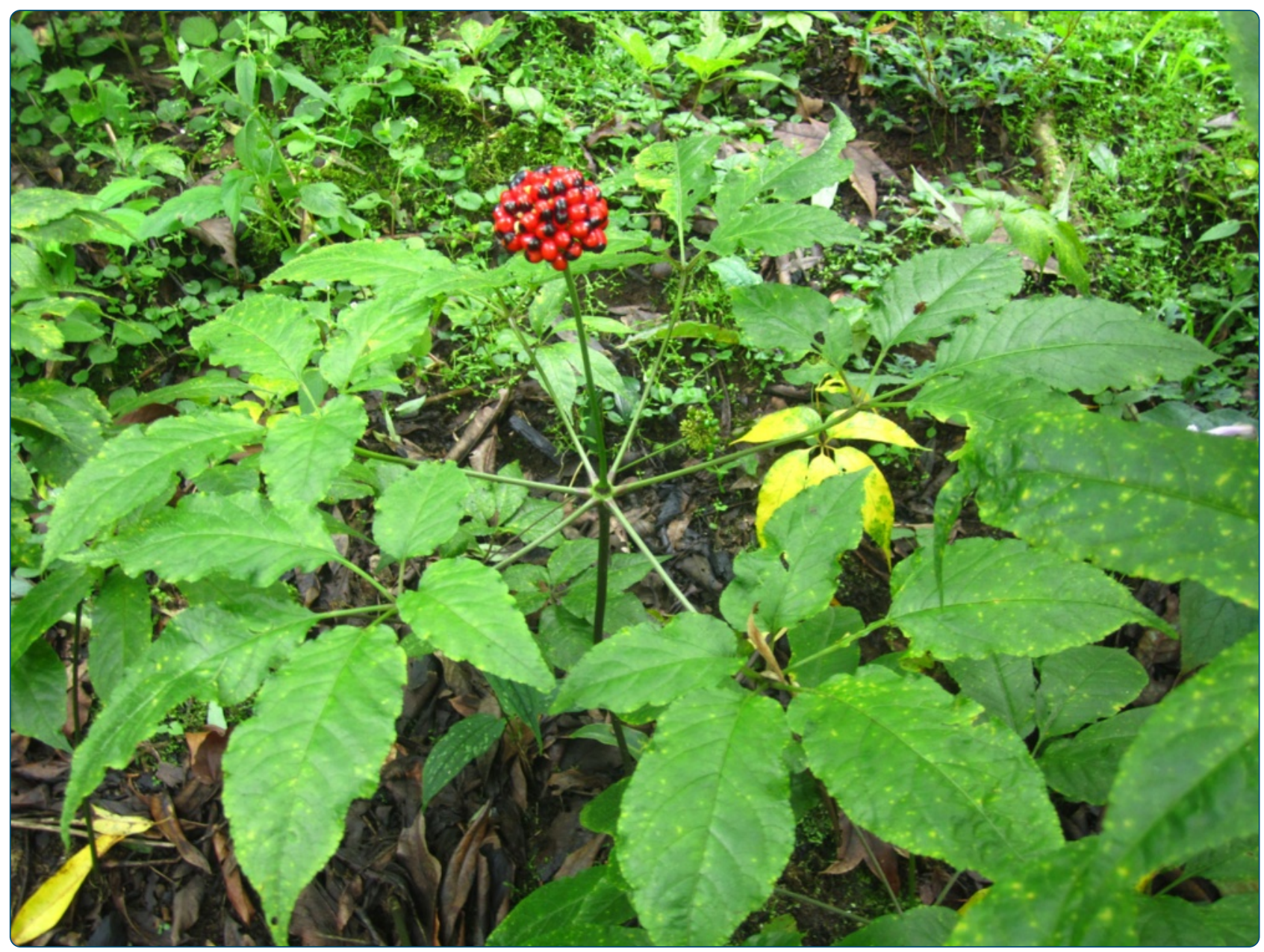

Transcriptome analysis of Panax vietnamensis var. fuscidicus discovers putative ocotillol-type ginsenosides biosynthesis genes and genetic markers

Zhang et al.

() Biomed Central 


\title{
Transcriptome analysis of Panax vietnamensis var. fuscidicus discovers putative ocotillol-type ginsenosides biosynthesis genes and genetic markers
}

Guang-Hui Zhang ${ }^{\dagger}$, Chun-Hua Ma ${ }^{\dagger}$, Jia-Jin Zhang, Jun-Wen Chen, Qing-Yan Tang, Mu-Han He, Xiang-Zeng Xu, $\mathrm{Ni-Hao}$ Jiang $^{*}$ and Sheng-Chao Yang ${ }^{*}$

\begin{abstract}
Background: $P$. vietnamensis var. fuscidiscus, called "Yesanqi" in Chinese, is a new variety of $P$. vietnamensis, which was first found in Jinping County, the southern part of Yunnan Province, China. Compared with other Panax plants, this species contains higher content of ocotillol-type saponin, majonoside $R_{2}$. Despite the pharmacological importance of ocotillol-type saponins, little is known about their biosynthesis in plants. Hence, P. vietnamensis var. fuscidiscus is a suitable medicinal herbal plant species to study biosynthesis of ocotillol-type saponins. In addition, the available genomic information of this important herbal plant is lacking.

Results: To investigate the $P$. vietnamensis var. fuscidiscus transcriptome, Illumina HiSeq ${ }^{\text {TM }} 2000$ sequencing platform was employed. We produced 114,703,210 clean reads, assembled into 126,758 unigenes, with an average length of 1,304 bp and N50 of 2,108 bp. Among these 126,758 unigenes, 85,214 unigenes (67.23\%) were annotated based on the information available from the public databases. The transcripts encoding the known enzymes involved in triterpenoid saponins biosynthesis were identified in our Illumina dataset. A full-length cDNA of three Squalene epoxidase (SE) genes were obtained using reverse transcription PCR (RT-PCR) and the expression patterns of ten unigenes were analyzed by reverse transcription quantitative real-time PCR (RT-qPCR). Furthermore, 15 candidate cytochrome P450 genes and 17 candidate UDP-glycosyltransferase genes most likely to involve in triterpenoid saponins biosynthesis pathway were discovered from transcriptome sequencing of $P$. vietnamensis var. fuscidiscus. We further analyzed the data and found 21,320 simple sequence repeats (SSRs), 30 primer pairs for SSRs were randomly selected for validation of the amplification and polymorphism in $13 \mathrm{P}$. vietnamensis var. fuscidiscus accessions. Meanwhile, five major triterpene saponins in roots of $P$. vietnamensis var. fuscidicus were determined using high performance liquid chromatography (HPLC) and evaporative light scattering detector (ELSD).
\end{abstract}

Conclusions: The genomic resources generated from $P$. vietnamensis var. fuscidiscus provide new insights into the identification of putative genes involved in triterpenoid saponins biosynthesis pathway. This will facilitate our understanding of the biosynthesis of triterpenoid saponins at molecular level. The SSR markers identified and developed in this study show genetic diversity for this important crop and will contribute to marker-assisted breeding for $P$. vietnamensis var. fuscidiscus.

Keywords: Panax vietnamensis var. fuscidicus, Transcriptome, Ginsenosides, Biosynthesis

\footnotetext{
*Correspondence: jnhskip@hotmail.com; shengchaoyang@163.com

†Equal contributors

Yunnan Research Center on Good Agricultural Practice for Dominant Chinese

Medicinal Materials, Yunnan Agricultural University, Kunming 650201,

Yunnan, People's Republic of China
}

\section{Biomed Central}

(c) 2015 Zhang et al.; licensee BioMed Central. This is an Open Access article distributed under the terms of the Creative Commons Attribution License (http://creativecommons.org/licenses/by/4.0), which permits unrestricted use, distribution, and reproduction in any medium, provided the original work is properly credited. The Creative Commons Public Domain Dedication waiver (http://creativecommons.org/publicdomain/zero/1.0/) applies to the data made available in this article, unless otherwise stated. 


\section{Background}

Ginsenosides are triterpenoid saponins found exclusively in Panax species belong to Araliaceae family. The Panax genus comprises approximately 14 species, more than 150 naturally occurring ginsenosides have been isolated from different parts of plants [1] and most of the saponins possess four types of aglycone moieties, i.e. protopanaxadiol, protopanaxatriol, ocotillol, and oleanolic acid types. The most widely used Panax species, such as $P$. ginseng, $P$. quinquefolium, and $P$. notoginseng mainly contain protopanaxadiol-type and protopanaxatriol-type saponins, the other species like $P$. japonicus and $P$. zingiberensis, contain a large amounts of oleanolic acid saponins [2,3], all of them do not have or only a small amount of ocotillol-type saponins. Up to now, only one species, $P$. vietnamensis have been found particularly accumulates surprisingly high content of ocotillol-type saponins, mainly majonoside $\mathrm{R}_{2}$, which is as high as $5.3 \%$ of the dried rhizome and exhibited anti-tumor and hepatocytoprotective activities [4-6].

2,3-oxidosqualene (OS), a precursor of terpenoids is synthesized via the mevalonic acid (MVA) pathway [7]. After the cyclization of 2,3-oxidosqalene by oxidosqualene cyclase (OSC), the triterpene skeletons are modified by hydroxylation and glycosidation that leads to the production of various ginsenosides, that are further catalyzed by cytochrome P450 monoxygenases (CYP450s) and uridine diphosphate (UDP)-dependent glycosyl-transferases (UGTs) [8] (Figure 1). The biosynthesis of protopanaxadiol, protopanaxatriol and oleanolic acid has been studied well, many genes involved in this pathway have been cloned and identified [9-17]. Recently, many putative triterpene saponin-biosynthetic genes in Panax species were detected using de novo sequencing and transcriptome analysis, especially in $P$. ginseng, $P$. quinquefolius, and $P$. notoginseng [18-22]. Despite the pharmacological importance of ocotillol-type saponins, little is known about their biosynthesis [1]. P. vietnamensis is the only species found in the narrow habitat in central Vietnam with high content of ocotillol-type saponins, which is also in the list of endanger species.

There are two kinds of pathways that form ocotillol. In pathway A, ocotillol might be biosynthesized via epoxidation of the double bond at C-24-C-25 of protopanaxatriol [1]. The enzyme catalyzes this reaction could be the ortholog of squalene epoxidase (SE) gene, because they epoxidized the similar double bonds of squalene or protopanaxatriol (Figure 1). In pathway B, OS is further epoxidized to 2,3 ; 22, 23- dioxidosqualene (DOS) by SE [23], followed by cyclization and hydroxylation to produce ocotillol, catalyzed by OSC and CYP450, respectively (Figure 1). In Arabidopsis, OSC, lupeol synthase (LUP1), directly converts DOS to oxacyclic triterpenoid epoxydammarane [23], so there might be similar OSC in
P. vietnamensis, which catalyzed the cyclization of DOS (Figure 1).

P. vietnamensis var. fuscidiscus, called "Yesanqi" in Chinese, is a new variety of $P$. vietnamensis, which was first found in Jinping County, the southern part of Yunnan Province, China [24]. P. vietnamensis var. fuscidiscus contains a higher content of majonoside $\mathrm{R}_{2}$ than other genotypes of $P$. vietnamensis [2]. Therefore, $P$. vietnamensis var. fuscidiscus is a perfect plant species for studying the biosynthesis mechanism of ocotillol-type saponins. Interestingly, $P$. vietnamensis var. fuscidiscus was also found in Yuanyang and Lvchun County, Honghe prefecture of Yunnan Province and some of them are found for more than 15 years and exhibited remarkable disease resistance under the high temperature and rainy conditions in this district, suggested that this specie could be used to improve disease resistance of $P$. notoginseng, an important cultivated Panax species in Yunnan Province of China.

Our goal of this study is to characterize the transcriptome of $P$. vietnamensis var. fuscidiscus using Illumina $\mathrm{HiSeq}^{\mathrm{TM}} 2000$ sequencing platform, to discover the candidate genes that encode enzymes in the triterpene saponin biosynthetic pathway, especially in ocotillol-type saponins biosynthesis, and produce information on SSR markers to facilitate the marker-assisted breeding of this species.

\section{Results and discussion}

\section{Illumina sequencing and de novo assembly}

$P$. vietnamensis var. fuscidiscus root tissue was used for transcriptome sequencing and analysis because root organs have been used for medicinal purpose. A cDNA library was constructed from total RNA of P. vietnamensis var. fuscidiscus roots, and sequenced using Illumina paired-end sequencing technology. After removal of adaptor sequences, ambiguous reads and low-quality reads $(\mathrm{Q} 20<20)$, a total of $114,703,210$ clean reads were obtained. The Q20 percentage (sequencing error rate $<1 \%$ ) and GC percentage were $97.23 \%$ and $43.25 \%$, respectively. An overview of the sequencing and assembly statistics are shown in Table 1. The high quality reads obtained in this study have been deposited in the NCBI SRA database (accession number: SRA146484).

All the clean reads $(114,703,210)$ were de novo assembled using the Trinity program into 161,443 contigs consisting of $218,944,221 \mathrm{bp}$. The size of the contigs ranged from 201 to $15,880 \mathrm{bp}$, with a mean length of 1,356 bp and N50 length of 2,087 bp. Among these contigs, $82,699(51.23 \%)$ were longer than $1000 \mathrm{bp}$, and 46,915 (29.06\%) contigs were shorter than 500 bp. Using paired-end joining and gap-filling methods, these contigs were further assembled into126,758 unigenes with an average length of $1,304 \mathrm{bp}$ and an N50 length of 


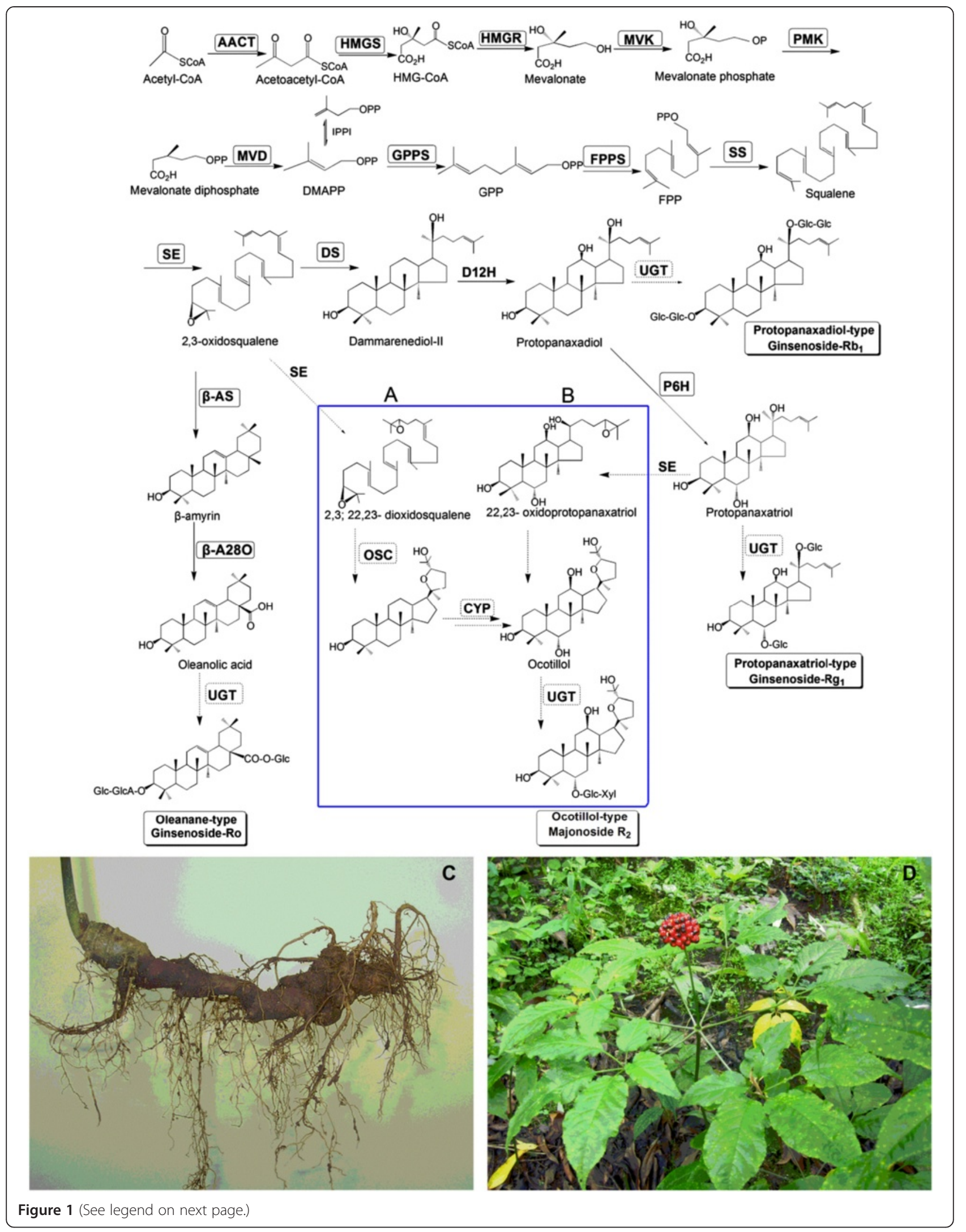


(See figure on previous page.)

Figure 1 Putative pathway for triterpene saponin biosynthesis. Putative pathway for triterpene saponin biosynthesis in $P$. vietnamensis var. fuscidicus. Two proposed pathways (A and B) for the biosynthesis of ocotillol-type saponins, mainlymajonoside $R_{2}$ in the horizontally grown rhizome (C) of $P$. vietnamensis var. fuscidicus (D). Enzymes found in this study are boxed. Abbreviations: AACT, acetyl-CoA acetyltransferase; $\beta$-AS, $\beta$-amyrin synthase; DMAPP, dimethylallyl diphosphate; DS, dammarenediol-Il synthase; FPP, farnesyl diphosphate; FPPS, farnesyl diphosphate synthase; Glc, glucose; GPP, geranyl pyrophosphate; GGPP, geranylgeranyl diphosphate; GGPPS, geranylgeranyl pyrophosphate synthase; GT, glycosyltransferase; HMG-CoA, 3-hydroxy-3-methylglutaryl coenzyme A; HMGR, HMG-CoA reductase; HMGS, HMG-CoA synthase; IPP, isopentenyl diphosphate; IPPI, IPP isomerase; MVD, mevalonate diphosphate decarboxylase; MVK, mevalonate kinase; P450, cytochrome P450; PMK, phosphomevalonate kinase; SE, squalene epoxidase; SS, squalene synthase.

2,108 bp. There were 60,741 unigenes (47.92\%) longer than $1,000 \mathrm{bp}$, and 28,676 unigenes (22.62\%) longer than 2,000 bp (Figure 2). In this study, the coding sequences (CDS) from all P. vietnamensis var. fuscidiscus unigene sequences were also detected and a total of 84,004 CDSs were obtained, among them, 24,580 CDSs (29.26\%) were longer than 1,000 bp (Figure 2).

To evaluate the quality of the assembled unigenes, all the usable sequencing reads were realigned to the unigenes using SOAPaligner [25], with up to 2 mismatches allowed. The sequencing depth ranged from 0.0173 to 50,534 fold, with an average of 50.67 fold. About $86.73 \%$ of the unigenes were realigned by more than 10 reads, $31.60 \%$ were supported by more than 100 reads, and $7.73 \%$ were supported by more than 1,000 reads (Additional file 1). In order to assess the extent of transcript coverage provided by unigenes and to evaluate how coverage depth affected the assembly of unigenes, we plotted the ratio of assembled unigene length to $P$. notoginseng orthologs length against coverage depth (Additional file 2A). Although many of the deeply sequenced $P$. vietnamensis var. fuscidiscus unigenes failed to cover the complete coding regions of their $P$. notoginseng orthologs, most of $P$. notoginseng orthologs coding

Table 1 Summary of Illumina Paired-end sequencing and assembly for $P$. vienamensis var. fuscidiscus

\begin{tabular}{lll}
\hline Database & Number & Total length(bp) \\
\hline Total Clean reads & $114,703,210$ & $11,470,321,000$ \\
Q20 percentage & $97.23 \%$ & \\
GC percentage & $43.25 \%$ & \\
Number of contigs & 161,443 & $218,944,221$ \\
Average length of contigs (bp) & 1,356 & \\
Max length of contigs (bp) & 15,880 & \\
Min length of contigs (bp) & 201 & \\
Contig size N50 (bp) & 2,087 & \\
Number of unigenes & $126,758,291,103$ \\
Average length of unigenes (bp) & 1,304 & \\
Max length of unigenes (bp) & 15,896 & \\
Min length of unigenes (bp) & 201 & \\
Unigene size N50 (bp) & 2,108 & \\
\hline
\end{tabular}

region can be covered by corresponding unigenes. To certain extent, increased coverage depth can result in higher coverage of the coding regions. The percentage of $P$. notoginseng orthologs coding sequence covered by all $P$. vietnamensis var. fuscidiscus unigenes was also performed. We found that 14,892 of the orthologs were covered by with a percentage of more than $80 \%$ and 3,252 of the orthologs were covered by unigenes with a percentage from $40 \%$ to $80 \%$. Furthermore, 326 orthologs were covered with only $20 \%$ or lower (Additional file 2B).

Due to the lack of $P$. vietnamensis var. fuscidiscus reference geneome availability, the reads produced by Illumina $\mathrm{HiSeq}^{\mathrm{rm}} 2000$ were assembled using the de novo assembler Trinity. In this study, the assembly results indicated that the length distribution pattern and mean length of contigs and unigenes was similar to those in the previous Illumina-transcriptome studies [26-28], suggesting that the transcriptome sequencing data from P. vietnamensis var. fuscidiscus are assembled well. Compared to previous transcriptomic studies in Panax species [18-22], we produced more numbers of unigenes, indicating that $P$. vietnamensis var. fuscidiscus genome is gene rich in comparison to Panax species.

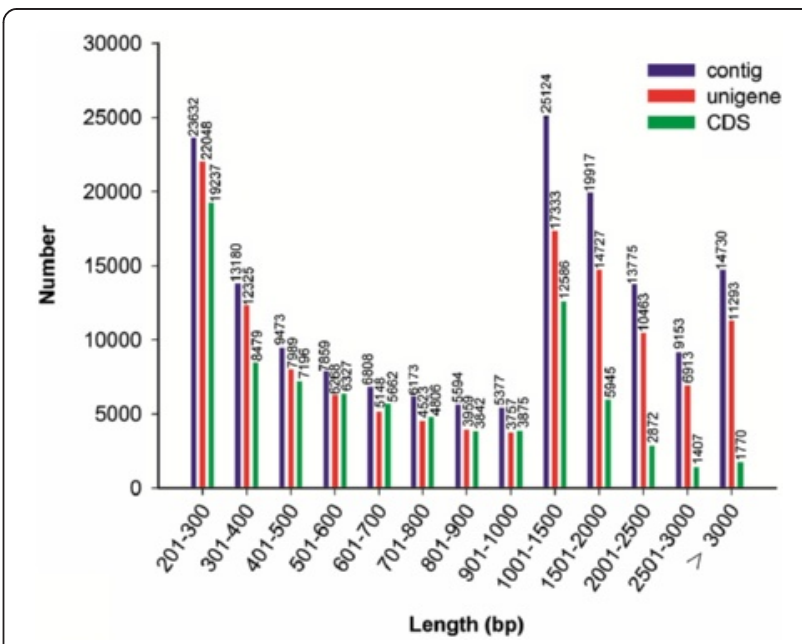

Figure 2 The length distribution of contigs, unigenes and CDSs. Overview of the $P$. vietnamensis var. fuscidiscus transcriptome assembly and the length distribution of the CDS. 


\section{Functional annotation}

A total of 85,214 unigenes $(67.23 \%)$ were annotated based on the information available from public databases including NCBI non-redundant protein $(\mathrm{Nr})$, Swiss-Prot protein, Cluster of Orthologous Groups (COG), and the Kyoto Encyclopedia of Genes and Genomes (KEGG) (Table 2). Among them, 16,602 unigenes showed significant matches to all four databases. Unigenes that were annotated as unique in public databases are as follows: 16,097 unigenes in the $\mathrm{Nr}$ database, 157 unigenes in the Swiss Prot database, 1 unigenes in the COG database, and 67 unigenes in the KEGG database (Additional file 3). Furthermore, about $32.77 \%$ of unigenes $(41,544)$ did not show any matches to known genes, these remaining unaligned unigenes may be considered as novel transcripts and specific genes from $P$. vietnamensis var. fuscidiscus.

Our results showed that approximately $95 \%$ of unigenes over 1,000 bp in length had BLAST matches against the $\mathrm{Nr}$ database, whereas only $41 \%$ of unigenes with lengths shorter than 1,000 bp generated BLAST matches (Additional file 4A). The same tendency was also observed in BLAST results against the SwissProt database (Additional file 4B). The e-value distribution of the top hits in the $\mathrm{Nr}$ database revealed that $62.34 \%$ of the mapped unigenes showed significant homology (evalue $<10^{-50}$ ), and $21.35 \%$ unigenes had high similarity (greater than $80 \%$ ) (Additional file $5 \mathrm{~A}$ and $\mathrm{C}$ ). The evalue and similarity distributions of the top hits in the Swiss-Prot database had a comparable pattern with $47.51 \%$ and $13.48 \%$ of the sequences possessing significant homology and similarity, respectively (Additional file $5 \mathrm{~B}$ and D). Our results also showed that $39.04 \%$ of the unigenes showed significant homology with gene sequences from Vitis vinifera (9,011, 19.07\%), followed by Arabidopsis thaliana (11.60\%), Glycine max (10.97\%), and Medicago truncatula (9.14\%) (Additional file 6).

\section{Gene ontology classification}

Based on the Nr annotation, Gene Ontology (GO) classification was used to classify the functions of all unigenes.

Table 2 Summary of the annotation percentage of $P$. vienamensis var. fuscidiscus as compared to public database

\begin{tabular}{lll}
\hline Database & $\begin{array}{l}\text { Number } \\
\text { of unigenes }\end{array}$ & $\begin{array}{l}\text { Annotation } \\
\text { percentage (\%) }\end{array}$ \\
\hline $\mathrm{Nr}$ & 84,983 & 67.04 \\
SwissProt & 66,471 & 52.44 \\
KEGG & 26,730 & 21.09 \\
COG & 34,918 & 27.55 \\
All annotated unigenes & 85,214 & 67.23 \\
Total unigenes & 126,758 & \\
\hline
\end{tabular}

A total of 43,163 unigenes were assigned to one or more gene ontology categories, 88,984 unigenes were from the cellular component, 79,483 unigenes from the biological process, and 51,542 unigenes from the molecular function. Under the biological process category: GO classification belongs to metabolic process $(21,803,27.43 \%)$, cellular process $(20,747,26.10 \%)$, and response to stimulus $(6,352,7.99 \%)$. In the cellular component group, unique sequences related to cell $(28,886,32.46 \%)$, cell part $(28,886,32.46 \%)$, organelle $(19,977,22.45 \%)$, and organelle part $(5,339,6.00 \%)$ were found. For the molecular function category, binding $(22,925,44.48 \%)$ and catalytic activity $(22.836,44.30 \%)$ represented the majority of unique sequences (Figure 3; Additional file 7).

\section{Conserved domain annotation and COG classification}

The conserved domains/families of the assembled unigenes encoding proteins were searched against the Pfam database (version 26.0) using Pfam_Scan program. A total of 3,602 conserved domains/families were identified from 46,649 unigenes $(36,80 \%$ of all unigenes) (Additional file 8). Among these protein domains/families, pentatricopeptide repeat domain (PPR) is the most abundant domain type, found in 2,822 unigenes. The PPR containing proteins are commonly found in the plants and although its function is still unclear, the PPR domain has been found in proteins involved in RNA editing in a number of recent studies [29-32]. Other highly represented domains/families were, WD repeat $(2,520$ unigenes), Protein kinase domain $(2,468$ unigenes), and Leucine Rich Repeat (2,449 unigenes). The WD repeat and Leucine Rich Repeat are involved in protein-protein interactions [33,34]. The role of protein kinase domain is found in signal transduction pathways, development, cell division, and metabolism in higher organisms $[35,36]$. Other domains identified abundantly included PPR repeat family (2,354 unigenes), RNA recognition motif (1,188 unigenes), Protein tyrosine kinase (1,035 unigenes), ABC transporter (488 unigenes), Mitochondrial carrier protein (462 unigenes), and Myblike DNA-binding domain (459 unigenes). For perspective, we have listed the top 20 most abundant protein domains/families in (Additional file 9).

All unigenes were subjected to a search against the COG database for functional prediction and classification. In total, 34,918 unigenes were annotated and grouped into 25 COG classifications. However, some of these unigenes were assigned to multiple COG classifications, altogether 63,521 COG functional annotations were obtained. Among the 25 COG categories, the cluster for general function prediction was the largest group $(11,382,17.92 \%)$, followed by replication, recombination and repair $(6,561,10.33 \%)$, transcription $(6,223,9.80 \%)$, 


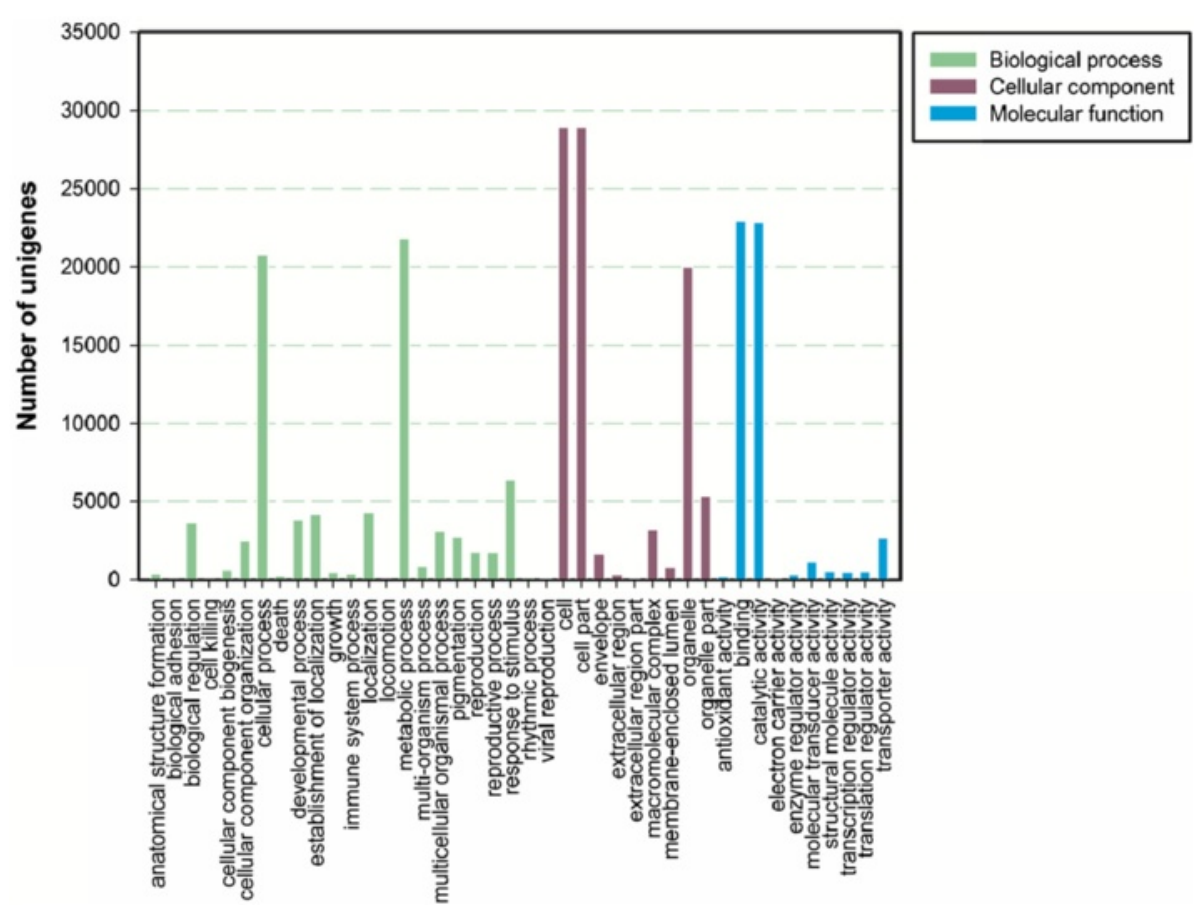

Figure 3 Gene Ontology classification of assembled unigenes. The unigenes were categorized into three main categories biological process, cellular component and molecular function.

and signal transduction mechanisms $(5,338,8.40 \%)$ (Figure 4).

\section{Functional classification by KEGG}

To elucidate the active biochemical pathways in $P$. vietnamensis var. fuscidiscus, unigenes were compared against the KEGG using BLASTx with an e-value $<1 \mathrm{e}^{-10}$ and the corresponding pathways were established. KEGG pathway analysis is helpful for predicting potential genes and their functions at a whole transcriptome level. A total of 26,730 unigenes $(21.09 \%)$ were annotated with KEGG and were assigned to 269 KEGG pathways (Additional file 10). RNA transport had the largest number of unigenes (825), followed by spliceosome (812 unigenes), protein processing in endoplasmic reticulum (697 unigenes), plant hormone signal transduction (684 unigenes), ubiquitin mediated proteolysis (679 unigenes), glycolysis/gluconeogenesis (636 unigenes), and purine metabolism (616 unigenes). KEGG metabolic pathways presented in our dataset include carbohydrate metabolism (3,809 unigenes), amino acid metabolism (2,352 unigenes), nucleotide metabolism (1,137 unigenes), lipid metabolism (2,023 unigenes), energy metabolism (1,782 unigenes), glycan biosynthesis

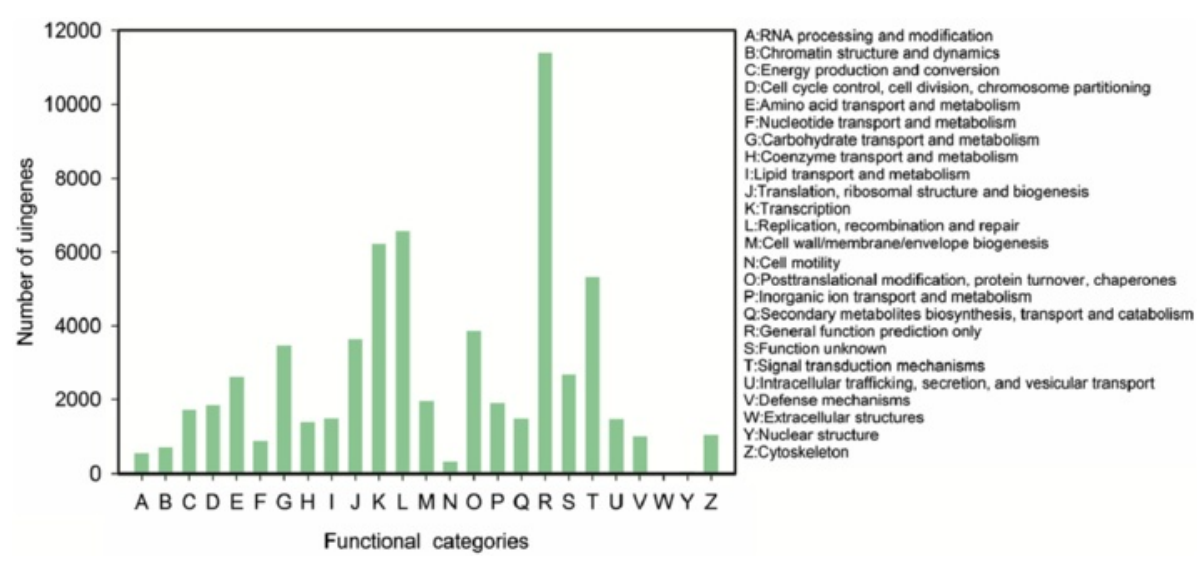

Figure 4 COG function classification of $P$. vienamensis var. fuscidiscus. 
and metabolism (1,282 unigenes), metabolism of cofactors and vitamins (1,053 unigenes), metabolism of other amino acids (710 unigenes), metabolism of terpenoids and polyketides (497 unigenes), biosynthesis of other secondary metabolites (457 unigenes), and xenobiotics biodegradation and metabolism (485 unigenes) (Figure 5A). In the metabolism of terpenoids and polyketides category, the most represented subcategories were terpenoid backbone biosynthesis (162 unigenes), followed by carotenoid biosynthesis (120 unigenes), limonene and pinene degradation (75 unigenes), tetracycline biosynthesis (36 unigenes), zeatin biosynthesis (30 unigenes), diterpenoid biosynthesis (23 unigenes), siderophore group nonribosomal peptides (19 unigenes), brassinosteroid biosynthesis (14 unigenes), geraniol degradation (11 unigenes), ansamycins biosynthesis (5 unigenes), and polyketide sugar unit biosynthesis (2 unigenes) (Figure 5B). These annotations will be a valuable resource for further research on specific pathways, structures and functions of genes in $P$. vietnamensis var. fuscidiscus.

\section{SSR marker discovery}

The potential SSRs were detected in all of the 126,758 assembled unigenes using MISA software. A total of 21,320 SSRs were identified in 17,780 unigenes (Table 3). Of all the SSR containing unigenes, 2,918 sequences contained more than one SSR, and 1,207 SSRs were present in compound form. The information of SSRs derived from all unigenes is shown in Additional file 11. Among these SSRs, the most frequent repeat motifs were di-nucleotides $(11,197,52.53 \%)$, followed by trinucleotides $(5,987,28.08 \%)$, tetra-nucleotides $(2,352$, $11.03 \%)$, penta-nucleotides $(924,4.33 \%)$, and hexanucleotides $(860,4.03 \%)$. Among the SSR with tandem repeats, SSRs with six tandem repeats $(5,657,26.53 \%)$ were most prevalent, followed by five tandem repeats $(3,988,18.71 \%)$, seven tandem repeats $(3,466,16.26 \%)$, and four tandem repeats $(3,356,15.74 \%)$ (Table 4). The di-nucleotide repeat AG/CT (26.7\%) was the most common motif, followed by the motif AT/AT (19.6\%), AAG/ CTT (7.5\%), and AC/GT (6.2\%). Our findings indicated that unigenes containing SSR markers were abundant in P. vietnamensis var. fuscidiscus. Based on those SSRs, 39,336 primer pairs were successfully designed using Primer3 (Additional file 12). The unigene derived markers generated in this study represent a valuable genetic resource for SSR mining and will aid future applications in research of this important herb crop.

\section{Validation of SSR markers}

Thirty SSR primer pairs were randomly selected and synthesized to evaluate the amplification efficiency and polymorphism in $13 \mathrm{P}$. vietnamensis var. fuscidiscus accessions from different countries and different genetic backgrounds. Twenty-nine (96.67\%) of the primer pairs successfully amplified clear and repeatable bands. Among the 29 successful primer pairs, 24 (80.00\%) primer pairs produced PCR amplicons at the expected size, and $4(13.33 \%)$ primer pairs generated PCR fragments longer than expected. We also found 15 (50.00\%) primer pairs exhibited polymorphisms (Additional file 13) among the $13 P$. vietnamensis var. fuscidiscus accessions. The observed number of alleles $(\mathrm{No})$ ranged from 0.25 to 0.52 , with an average value of 0.42 ; the effective number of alleles $(\mathrm{Ne})$ ranged from 0.20 to 0.41 , with a mean level of 0.34; Shannon's information index $(I)$ varied from 0.16 to 0.33 , with an average value of 0.27 ; the number of polymorphic loci $(N P)$ ranged from 0.80 to 4.40 , with a mean level of 2.5 ; the percentage of polymorphic loci $(P P B)$ ranged from 8.89 to 48.89 , with a mean level of 28.15; and polymorphism information content (PIC) values ranged from 0.29 to 0.50 with an average of 0.44 . These results indicated that there is a good genetic diversity existed among $13 P$. vietnamensis var. fuscidiscus accessions.

The dendrogram constructed based on UPGMA (unweighted pair group method with arithmetic average) clustering method was used to perform genetic correlation analysis among the 13 P. vietnamensis var. fuscidiscus accessions (Figure 6). The coefficients of genetic similarity among the 13 P. vietnamensis var. fuscidiscus accessions ranged from 0.82 to 0.94 , indicating a high genetic similarity among them. UPGMA cluster analysis grouped these individuals into two groups at the similarity level of 0.836 . According to the dendrogram, all the 3 accessions from Laos were clustered into cluster I. In cluster II, all the 10 accession of $P$. vietnamensis var. fuscidiscus from China were clustered into one group. The results of the cluster analysis showed that the individuals from the same area tend to clustered together. Therefore, the UPGMA cluster analysis based on SSR data was closely related to the geographical origins. Meanwhile, these results demonstrate that SSRs primer pairs derived from $P$. vietnamensis var. fuscidiscus unigenes can distinguish varieties without morphological diversities, and will be a powerful tool for genetic applications in this herb crop.

\section{Candidate genes encoding enzymes involved in ginsenosides biosynthesis}

The transcripts encoding all the known enzymes involved in triterpenoid saponin pathway were discovered from this Illumina transcriptome dataset, including AACT (acetyl-CoA acetyltransferase), HMGS (HMGCoA synthase), HMGR (HMG-CoA reductase), MVK (mevalonate kinase), PMK (phosphomevalonate kinase), MVD (mevalonate diphosphate decarboxylase), GGPPS 

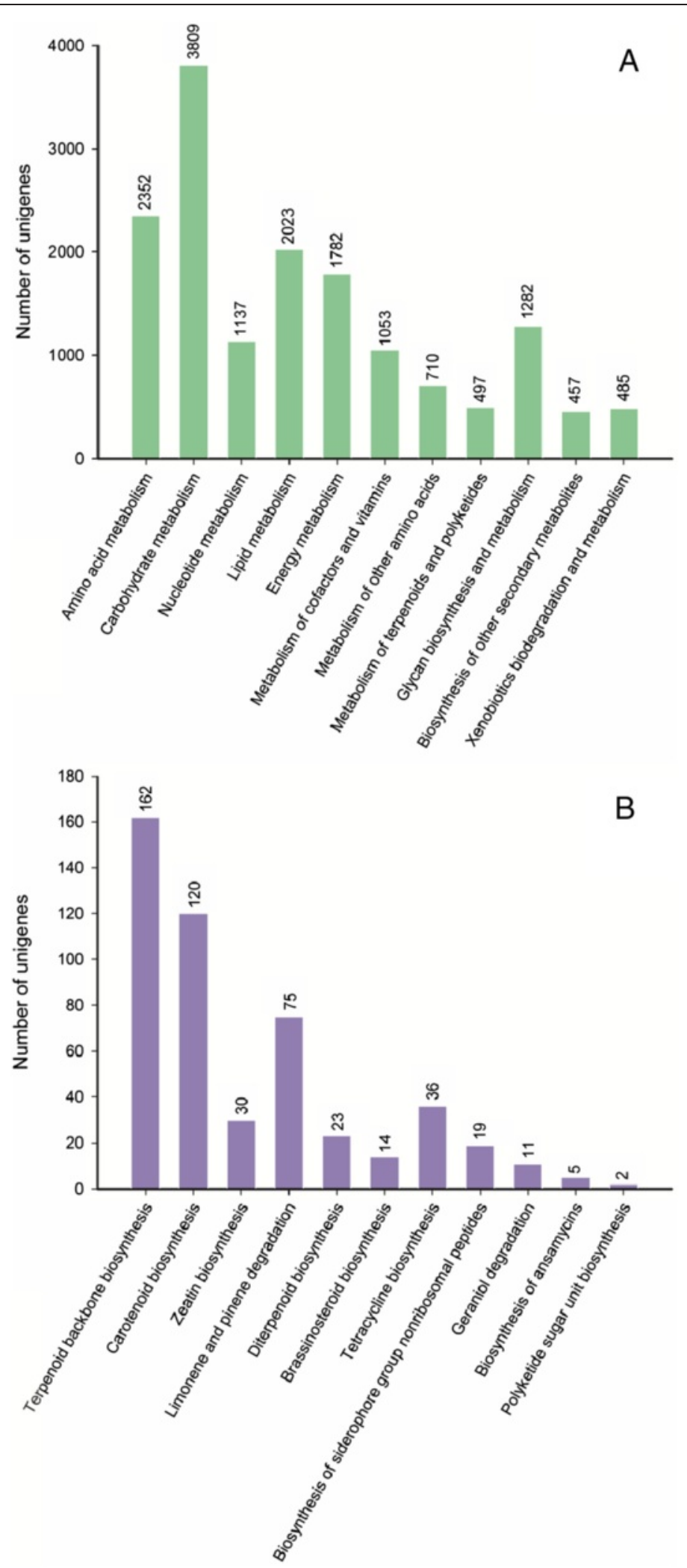

Figure 5 Pathway assignment based on KEGG. (A) Classification based on metabolism categories; (B) Classification based on metabolism of terpenoids and polyketides. 
Table 3 Summary of SSR searching results

\begin{tabular}{ll}
\hline Item & Number \\
\hline Total number of sequences examined & 126,758 \\
Total size of examined sequences (bp) & $165,291,103$ \\
Total number of identified SSRs & 21,320 \\
Number of SSR containing Sequences & 17,780 \\
Average number of SSRs per 10 kb & 1.29 \\
Number of sequences containing more than 1 SSR & 2,918 \\
Number of SSRs present in compound formation & 1,207 \\
\hline
\end{tabular}

(geranylgeranyl pyrophosphate synthase), FPPS (farnesyl diphosphate synthase), IPPI (isopentenyl diphosphate isomerase), SS (squalene synthase), SE, $\beta$-AS ( $\beta$-amyrin synthase), DS (dammarenediol-II synthase), D12H (dammarenediol 12-hydroxylase), $\mathrm{P} 6 \mathrm{H}$ (protopanaxadiol 6hydroxylase), and $\beta$-A28O ( $\beta$-amyrin 28 -oxidase) (Table 5, Additional file 14). The discovery of many genes related to triterpenoid pathway may help us to investigate the cause of high content of protopanaxadioltype and protopanaxatriol-type saponins, such as Rb1, $\mathrm{Rd}$ and $\mathrm{Rg}_{1}$ in $P$. vietnamensis var. fuscidiscus.

Majonoside $R_{2}$ is the main ginsenoside in $P$. vietnamensis var. fuscidiscus, so we focused on the discovery of the putative genes that might be involved in ocotilloltype ginsenoside biosynthesis. As mentioned above, the formation of ocotillol needs SE and OSC with "new" functions. Generally, SE catalyzes the epoxidation of squalene to OS in terpenoid biosynthesis, but in $P$. vietnamensis var. fuscidicus, SE might catalyze the epoxidation of terminal olefin of protpanaxatriol or OS (Figure 1). Moreover, 15 unigenes matched to SE of other plants were discovered in our Illumina dataset. Using the primers designed based on the sequences of these SE unigenes, a full-length cDNA of three SE genes were obtained using reverse transcription PCR (RTPCR), named PvfSE1, PvfSE2, and PvfSE3, respectively (GenBank: KJ946467, KJ946468 and KJ946469). The three cloned $S E$ genes may play different roles in sterol or ginsenoside biosynthesis in this new variant (data not shown), and a series of relevant studies are currently underway to determine the function of them. Many genes encoding OSCs have been isolated in plants; including those encode $\beta$-AS, DS, lupeol synthase (LUS), and cycloartenol synthase (CAS) [16,37-39]. Except for $\beta$-AS and DS, no unigene matched to LUS and CAS was found (Table 5). Characterizing the functions of these unigenes will help us for understanding the molecular mechanism of biosynthesis of ocotillol-type ginsenoside.

\section{The cytochrome P450 monooxygenases and UDP-glycosyltransferase genes}

Identification of specific CYP450 enzymes responsible for the production of particular metabolites is difficult due to its large numbers [40]. However, only a few CYP450s have been identified in plants, which involved in triterpenoid saponins biosynthesis. The CYP716A subfamily members in $M$. truncatula (CYP716A12) and V. vinifera (CYP716A15 and CYP716A17) are multifunctional oxidases, with $\beta$-A28O, $\alpha-$ amyrin 28-oxidase and lupeol 28-oxidase activities [41,42]. In $P$. ginseng, three CYP716A subfamily members have been isolated and characterized functionally, encode $\mathrm{P} 6 \mathrm{H}$ (CYP716A47), D12H (CYP716A53v2) and $\beta$-A28O (CYP716A52v), respectively [13-15]. Licorice (Glycyrrhiza uralensis) CYP88D6 catalyze C-11 oxidation of $\beta$-amyrin in glycyrrhizin biosynthesis [43], while GuCYP72A154 and $M$. truncatula CYP72A63 catalyze C-30 oxidation of $\beta$-amyrin [44]. Both G. $\max$ CYP93E1, licorice CYP93E2 and CYP93E3 catalyze the $C$-24 hydroxylation of $\beta$-amyrin and sophoradiol in soyasaponin biosynthesis $[43,45,46]$. Oat (Avena strigosa) CYP51H10 is able to catalyze both hydroxylation and epoxidation of $\beta$-amyrin to produce $12,13 \beta$ epoxy-3 $\beta, 16 \beta$-dihydroxy-oleanane [47-49]. Arabidopsis CYP708A2 and CYP705A5 were identified as a thalinol hydroxylase and thaliana-diol desaturase, respectively [50]. $M$. truncatula CYP72A61v2 and CYP72A68v2 catalyze C-22 of 24-OH- $\beta$-amyrin and $\mathrm{C}-23$ of oleanolic acid, respectively [46].

For discovering the candidate CYP450s involved in ginsenosides biosynthesis in the transcriptomic data of $P$. vietnamensis var. fuscidicus, 251 unigenes which is annotated to

Table 4 Distribution of identified SSRs using the MISA software

\begin{tabular}{|c|c|c|c|c|c|c|c|c|c|c|c|}
\hline \multirow[t]{2}{*}{ Motif } & \multicolumn{9}{|c|}{ Repeat numbers } & \multirow[t]{2}{*}{ Total } & \multirow[t]{2}{*}{$\%$} \\
\hline & 4 & 5 & 6 & 7 & 8 & 9 & 10 & 11 & $\geq 11$ & & \\
\hline Di- & 0 & 0 & 4,030 & 2,480 & 1,576 & 1,240 & 1,230 & 627 & 14 & 11,197 & 52.52 \\
\hline Tri- & 0 & 3,433 & 1,448 & 966 & 126 & 2 & 0 & 3 & 9 & 5,987 & 28.08 \\
\hline Tetra- & 1,789 & 446 & 96 & 12 & 0 & 6 & 0 & 0 & 3 & 2,352 & 11.03 \\
\hline Penta- & 812 & 94 & 10 & 7 & 0 & 0 & 0 & 0 & 1 & 924 & 4.33 \\
\hline Hexa- & 755 & 15 & 73 & 1 & 9 & 0 & 4 & 2 & 1 & 860 & 4.03 \\
\hline Total & 3,356 & 3,988 & 5,657 & 3,466 & 1,711 & 1,248 & 1,234 & 632 & 28 & 21,320 & 100 \\
\hline$\%$ & 15.74 & 18.71 & 26.53 & 16.26 & 8.03 & 5.85 & 5.79 & 2.96 & 0.13 & 100 & \\
\hline
\end{tabular}




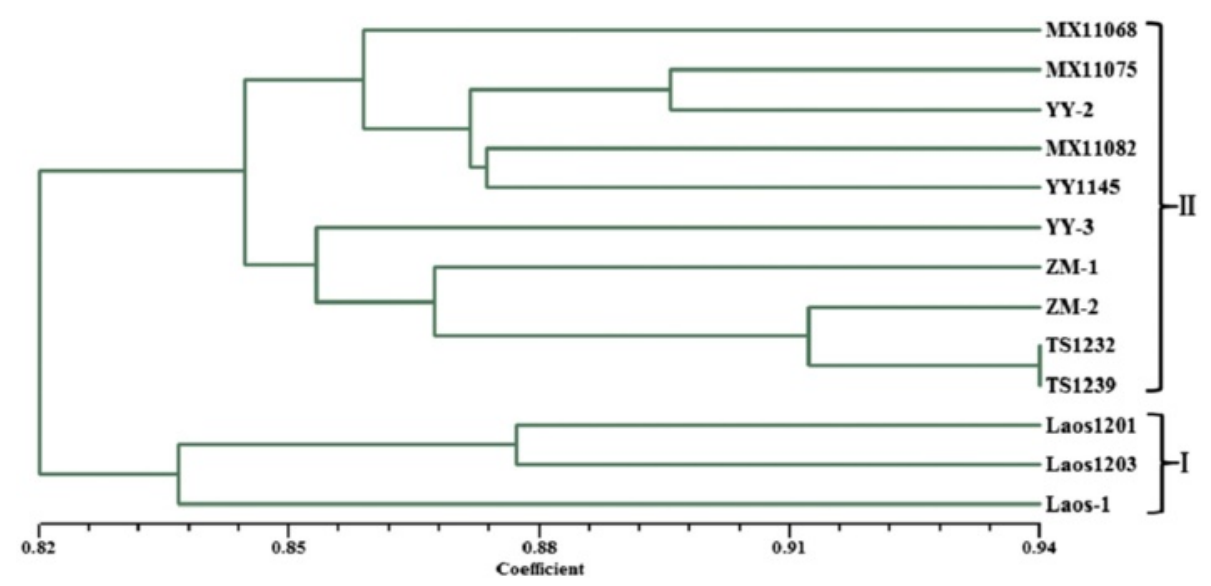

Figure 6 UPGMA dendrogram of 13 accessions of $P$. vienamensis var. fuscidiscus. Dendrogram constructed with UPGMA clustering method among 13 different accessions of $P$. vienamensis var. fuscidiscus.

be CYP450 (Additional file 15) were compared with CYP450s mentioned above. As shown in Figure 7, the orthologous genes of PgCYP716A52v2 (unigene 0046586), PgCYP716A53v2 (unigene0016477), and PgCYP716A47 (unigene0036796, unigene0036797, unigene0036795, and unigene0036798) were found. Besides, one unigene (unigene0027571) is also belong to CYP716A subfamily, indicate this unigene may has different functions from other CYP716A subfamily in $P$. ginseng. Furthermore, 4 unigenes (unigene0044841, unigene0044844, unigene0145860, and unigene0006105) are highly homologous to $A$. thaliana thalianol hydroxylase (AtCYP708A2). Unigene0038 is homologous to AsCYP51H10, unigene0042080 is homologous to GuCYP93E3 and AtCYP705A5, unigene0039295 and unigene0039296 are homologous to MtCYP72A67v2 and MtCYP72A8v2 (Figure 7). Two unigenes (unigene0039295 and unigene0039296) are the orthologous gene of 11-oxo$\beta$-amyrin 30-oxidase and highly homologous to GuCYP72A154, MtCYP72A63, MtCYP72A61v2, and MtCYP72A68v2 [44,46]. Unigene00402080 is highly homologous to GuCYP93E3 and MtCYP93E2 [43,51], may encode enzyme catalyze $\mathrm{C}$ - 24 hydroxylation of $\beta$-amyrin.

UGTs catalyze the glucosylation of C-3, C-12, C-20 hydroxyl, and C28-carboxyl for the biosynthesis of ginsenosides in P. vietnamensis var. fuscidicus. Even though UGTs catalyze the last committed step of ginsenoside biosynthesis; no UGT was functionally characterized from Panax species, only one putative UGT gene

Table 5 Transcripts involved in triterpene saponin biosynthesis in $P$. vietnamensis var. fuscidiscus

\begin{tabular}{lll}
\hline Gene name & EC number & Unigene number \\
\hline AACT, acetyl-CoA acetyltransferase & 2.3 .1 .9 & 8 \\
HMGS, hydroxymethylglutaryl-CoA synthase & 2.3 .3 .10 & 7 \\
HMGR, hydroxymethylglutaryl-CoA reductase & 1.1 .1 .34 & 4 \\
MVK, mevalonate kinase & 2.7 .1 .36 & 3 \\
PMK, phosphomevalonate kinase & 2.7 .4 .2 & 10 \\
MVD, mevalonate diphosphate decarboxylase & 4.1 .1 .33 & 2 \\
GGPPS, geranylgeranyl pyrophosphate synthase & 2.5 .1 .29 & 64 \\
FPPS, farnesyl diphosphate synthase & 2.5 .1 .10 & 34 \\
IPPI, isopentenyl diphospate isomerase & 5.3 .3 .2 & 1 \\
SS, squalene synthase & 2.5 .1 .21 & 6 \\
SE, squalene epoxidase & 1.14 .99 .7 & 15 \\
DS, dammarenediol-II synthase & 4.2 .1 .125 & 1 \\
$\beta$-AS, $\beta$-amyrin synthase & 5.4 .99 .39 & 11 \\
$\beta$-A28O, $\beta$-amyrin 28-oxidase (CYP716A52v2 in P. ginseng) & $1.14 .13 .-$ & 1 \\
D12H, dammarenediol 12-hydroxylase (CYP716A47 in P. ginseng) & 1.14 .13 .183 & 4 \\
P6H, protopanaxadiol 6-hydroxylase (CYP716A53v2 in P. ginseng) & 1.14 .13 .184 & 1 \\
\hline
\end{tabular}




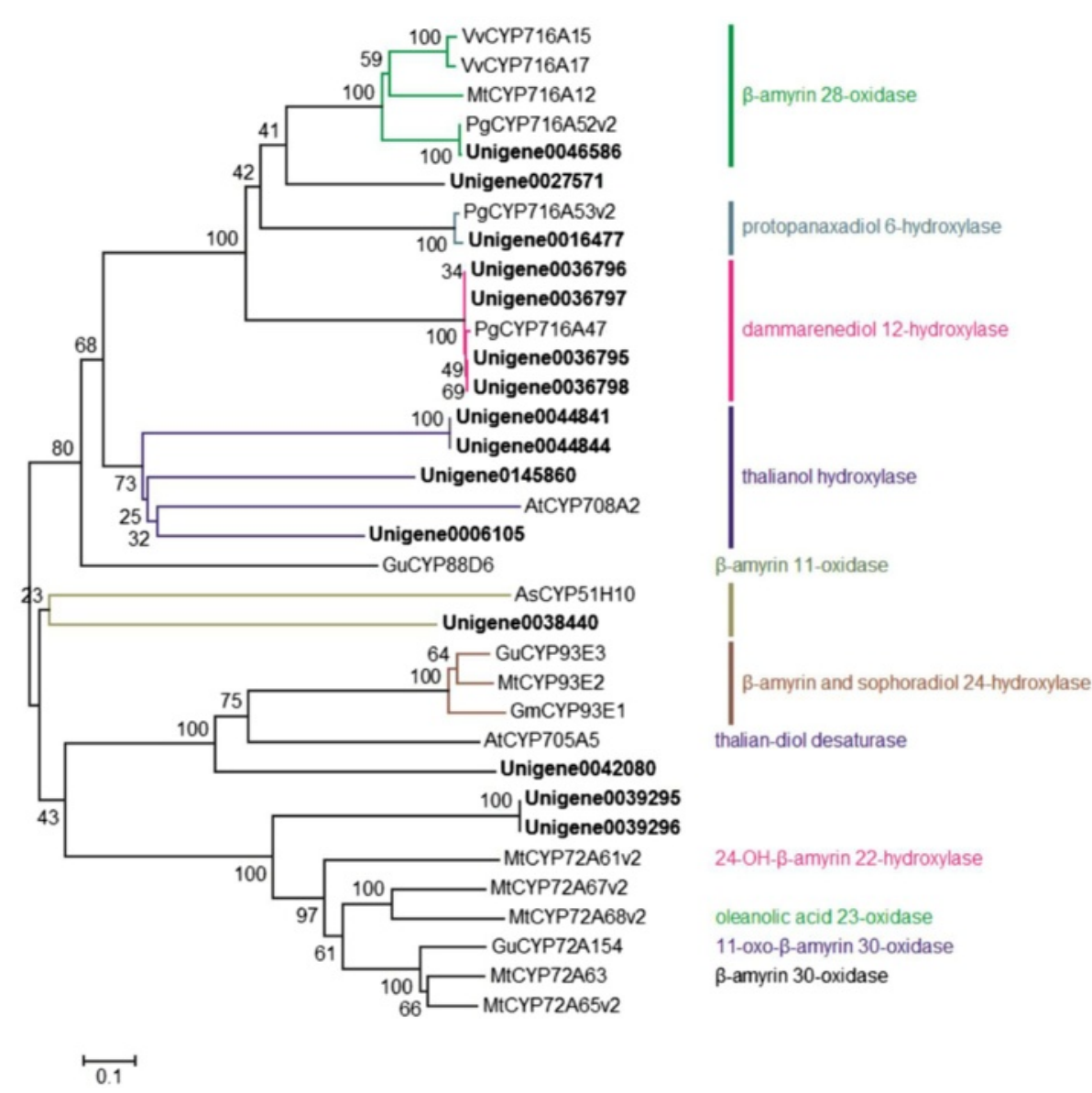

Figure 7 Phylogenetic tree of CYP450s. Phylogenetic tree of the P. vienamensis var. fuscidiscus CYP450s. Phylogenetic tree constructed based on the deduced amino acid sequences for the P. vienamensis var. fuscidiscus CYP450s (bold letters) and other plant CYP450s involved in triterpenoid biosynthesis. Protein sequences were retrieved from NCBI GenBank using the following accession numbers: Vitis vinifera VvCYP716A15, (BAJ84106.1) and VVCYP716A17 (BAJ84107.1); Medicago truncatula MtCYP716A12, (ABC59076.1), MtCYP93E2 (ABC59085), MtCYP72A63 (H1A981.1), MtCYP72A65V2, (BAL45202), MtCYP72A67V2 (BAL45203) and MtCYP72A68V2 (BAL45204), and MtCYP72A61V2 (BAL45199); Panax ginseng PgCYP716A52v2 (AFO63032.1), PgCYP716A53V2 (17CT85.1) and PgCYP716A47 (H2DH16.2); Arabidopsis thaliana AtCYP708A2 (NP_001078732.1) and AtCYP705A5 (EFH40098); Glycyrrhiza uralensis GuCYP88D6 (B5BSX1.1), GuCYP93E3 (BAG68930) and GuCYP72A154 (H1A988.1); Avena strigosa AsCYP51H10 (ABG88965.1); Glycine max GmCYP93E1 (NP_001236154.1).

(PnUGT1) was cloned from P. notoginseng [52], which had relative close relationship to the triterpene UDPglucosyltransferase of $M$. truncatula UGT71G1 [53]. In cDNA library of $P$. vietnamensis var. fuscidicus, 282 unigenes were found to encode UGTs (Additional file 16). The phylogenetic relationship between UGTs from $P$. vietnamensis var. fuscidicus and characterized UGTs from other plants was depicted in Figure 8. Except the orthologous genes of PnUGT1 (unigene0045236), unigene0071620 is highly homologous to Barbarea vulgaris UGT73C11 and UGT73C10, which catalyze sapogenin 3-O-glucosylation [54], suggested that unigene 0071620 has the same function in P. vietnamensis var. fuscidicus. Besides, unigene005064, unigene0031030, and unigene0031036 have close relationship to Solanum aculeatissimum steroidal saponin UDPglucosyltransferase SaGT4A [55], M. truncatula UGT73F3 [56], MtUGT73K1, MtUGT71G1 [53], and soybean
UGT73F4 [57], indicated that those unigenes are also involved in ginsenoside biosynthesis. Furthermore, 2 unigenes (unigene0063740 and unigene0063744) have close relationship to Saponaria vaccaria UGT74M1, which is a triterpene carboxylic acid glucosyltransferase [58], suggested that these unigenes may catalyze the glucosylation of C28-carboxyl for the biosynthesis of ginsenoside Ro.

\section{RT-qPCR analysis of the ginsenoside synthesis related genes}

The RT-qPCR analysis was used to investigate the tissue-specific expression patterns of 10 unigenes related to ginsenoside biosynthesis in this species. The expression pattern of these genes is shown in Figure 9. The unigenes encoding HMGS, MVK, MVD, and IPPI were expressed at much higher level in young stems than in other tissues (lateral roots, root and leaves). The gene 


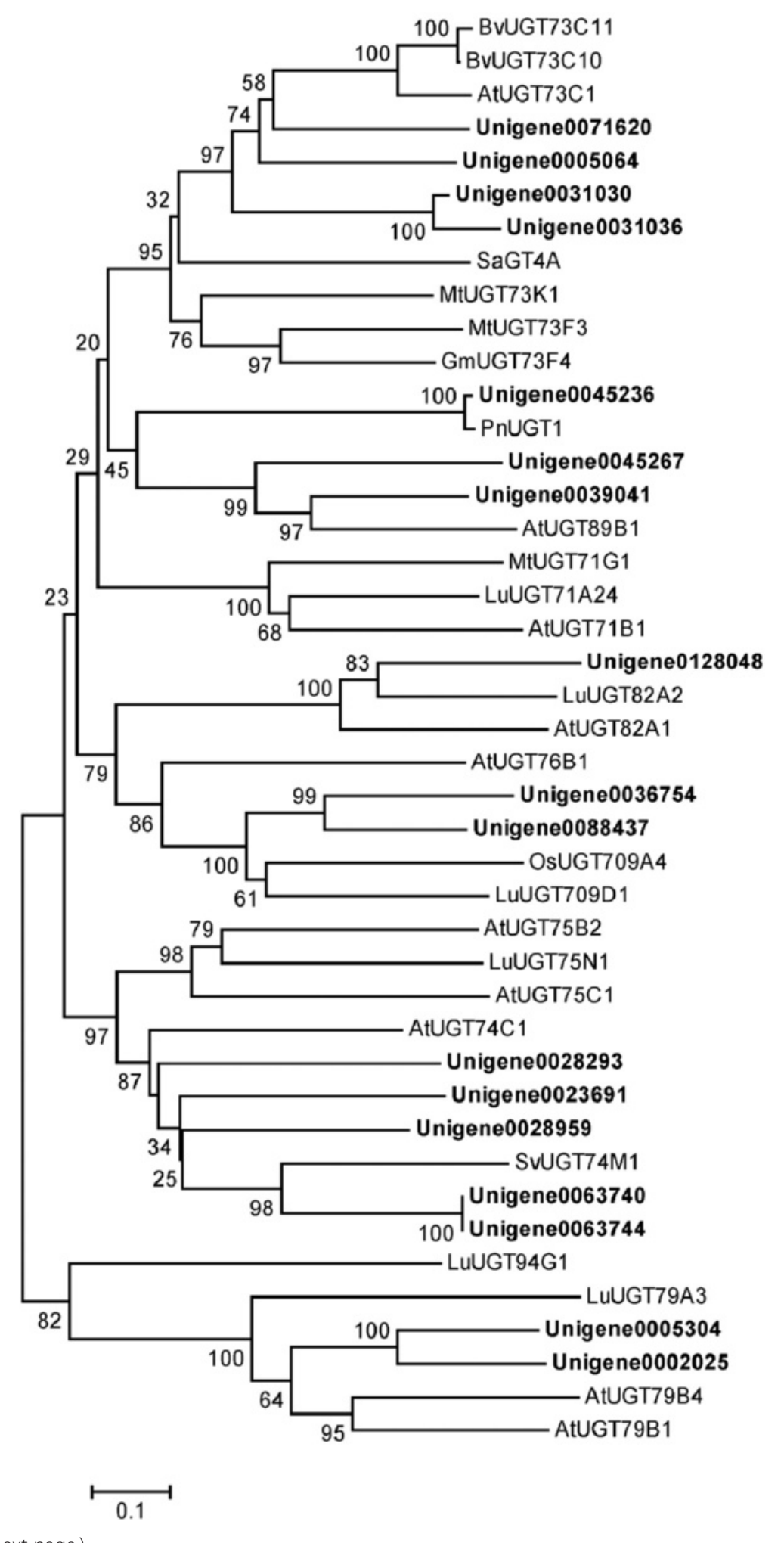

Figure 8 (See legend on next page.) 
(See figure on previous page.)

Figure 8 Phylogenetic tree of UGTs. Phylogenetic tree constructed based on the deduced amino acid sequences for the $P$. vienamensis var. fuscidiscus UGTs (bold letters) and other plant UGTs. Accession numbers in the NCBI GenBank database are as follows: Barbarea vulgaris BvUGT73C11 (AFN26667) and BvUGT73C10 (AFN26666); Arabidopsis thaliana AtUGT73C1 (NP_181213.1), AtUGT82A1 (NP_188864.1), AtUGT76B1 (NP_187742.1), AtUGT71B1 (NP_188812.1), AtUGT89B1 (NP_177529.2), AtUGT75B2 (NP_172044.1), AtUGT75C1 (NP_193146.1), AtUGT74C1 (NP_180738.1), AtUGT79B4 (Q9LJA6.1) and AtUGT79B1 (Q9LWW3.1); Solanum aculeatissimum SaGT4A (BAD89042); Medicago truncatula MtUGT73K1 (AAW56091), MtUGT73F3 (ACT34898) and MtUGT71G1 (AAW56092); Glycine max GmUGT73F4 (BAM29363); Panax notoginseng PnUGT1 (JX018210); Oryza sativa OsUGT709A4 (Q7XHR3); Saponaria vaccaria SvUGT74M1 (ABK76266); Linum usitatissimum LuUGT71A24 (AFJ52909), LuUGT82A2 (AFJ52979), LuUGT709D1 (AFJ53007), LuUGT75N1 (AFJ52962), LuUGT94G1 (AFJ53037.1), LuUGT79A3 (AFJ52973.1).

encoding SS was highly expressed in the leaves and stems. The HMGR gene showed very high expression in the leaf tissue. All genes mentioned above play a role in upstream biochemical reactions of the ginsenoside pathway, and showed high expression in leaves and young stems, which indicates that leaves and young stems are the main factories for synthesizing the precursors of ginsenosides. SE gene was involved in the formation of 2,3-oxidosqualene, a precursor of various ginsenosides. To further identify the potential candidates from SE homologs involved in ginsenoside biosynthesis, the expression levels of three putative SE genes (SE1, SE2, and SE3) in different organs were analyzed. SE1 and SE3 genes were expressed much higher in young stems and
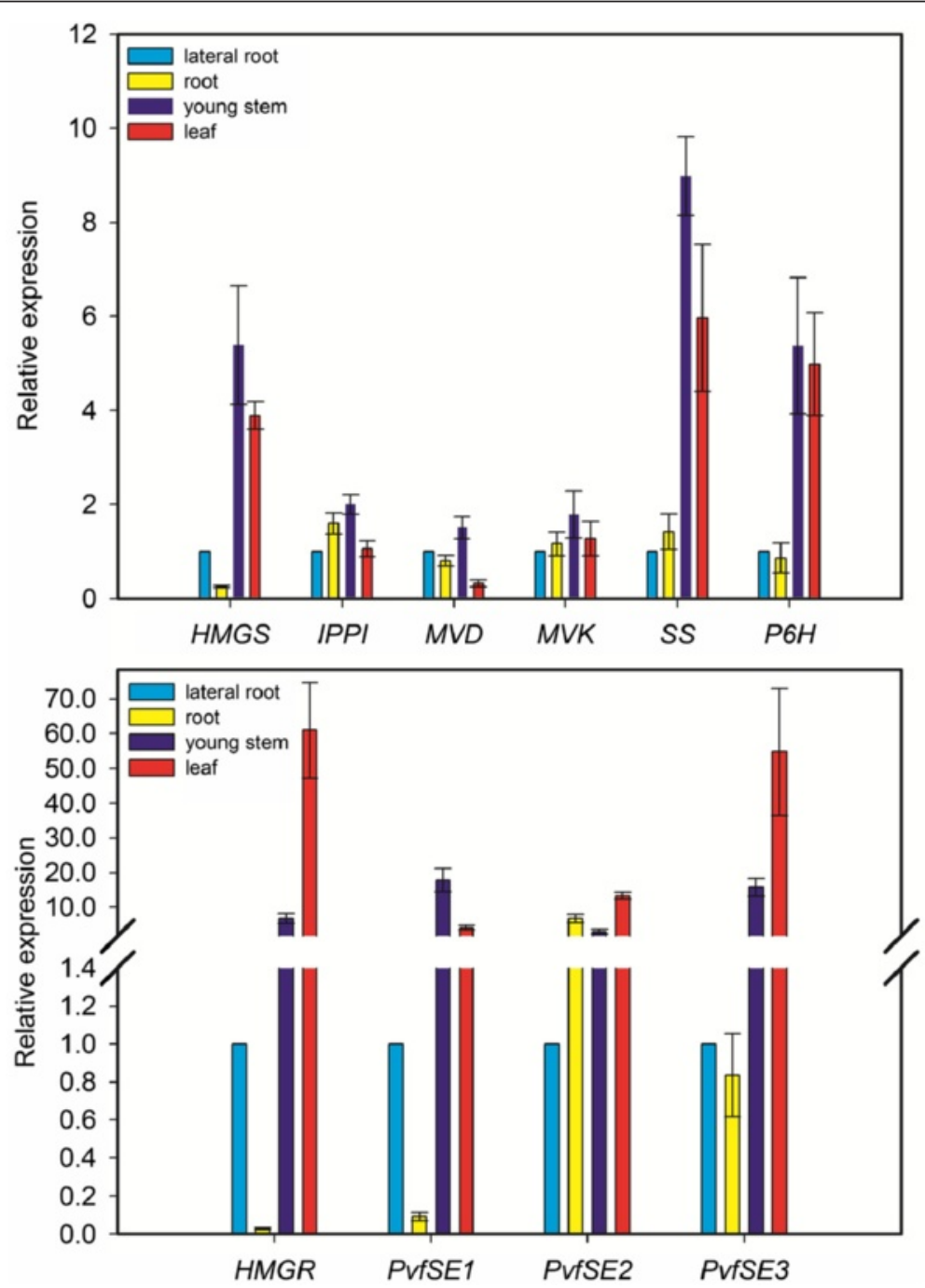

Figure 9 qRT-PCR analysis of unigenes involved in triterpene saponin biosynthesis. Validation of candidate $P$. vietnamensis var. fuscidiscus unigenes involved in triterpene saponin biosynthesis by qRT-PCR. Bars represent the mean ( \pm SD) of four experiments. 
leaves than in other tissues, respectively (Figure 9). Whereas the expression level of SE2 was higher in the roots as compared to that of SE1 and SE3. These three putative $S E$ genes have different expression patterns in different tissues, similar to what was found in previous studies [59]. Thus, we supposed that these three putative SE genes play different roles in ginsenosides biosynthesis. The gene encoding P6H was highly expressed in leaves and young stems than in roots and hairy roots. The $\mathrm{P} 6 \mathrm{H}$ was predicted to catalyze protopanaxadiol to protopanaxatriol. A higher expression of $\mathrm{P} 6 \mathrm{H}$ observed in leaves and young stems but protopanaxatriol-type ginsenosides accumulated mainly in roots and hairy roots, again indicating that leaves and young stems were the main synthesis site of the triterpene skeletons. The results demonstrate that several genes involved in ginsenoside biosynthesis showed diverse expression patterns in different tissues. The analysis of the expression patterns of these genes in different tissues will be helpful to further understand the mechanism of ginsenoside biosynthesis.

\section{Quantitative analysis of five major triterpene saponins in roots of $P$. vietnamensis var. fuscidicus}

The content of main component is the most widely used indicator to measure the quality of herb, so quantitative analysis of main component has important practical significance. According to previous research [2], majonoside $\mathrm{R} 2$, ginsenoside $\mathrm{Rg} 1, \mathrm{Rb} 1, \mathrm{Rd}$ and notoginsenoside $\mathrm{R} 1$ are considered as the five main components of $P$. vietnamensis var. fuscidicus. Herein, the content of five major triterpene saponins in roots of $P$. vietnamensis var. fuscidicus was determined. High performance liquid chromatography with evaporative light scattering detector (HPLC-ELSD) was employed for quantitative analysis of majonoside R2, due to its low UV absorptivity. As shown in Figure 10A and B, the peak of majonoside $\mathrm{R} 2$ was identified by direct comparing the retention times of the peaks with those of the standard majonoside R2 eluted under the same conditions. The content of majonoside R2 in in roots of $P$. vietnamensis var. fuscidicus is about $68 \mathrm{mg} / \mathrm{g}$, indicated that majonoside R2 were rich in the roots of this species. Quantitative analysis of other four triterpene saponins in the roots of this herb were performed using high performance liquid chromatography (HPLC). As shown in Figure 10C and D, the investigated saponins were well separated within $55 \mathrm{~min}$. The content of ginsenoside Rg1, Rb1, notoginsenoside R1, and ginsenoside $\mathrm{Rd}$ in the roots of this herb were approximately $52.7,17.9,17.8$ and $3.2 \mathrm{mg} / \mathrm{g}$, respectively. The above results were approximately in accordance with previous studies [2], indicated that our quantitative results are reliable. We believe that these data will be useful for pharmacological evaluation and quality control of this new variety.

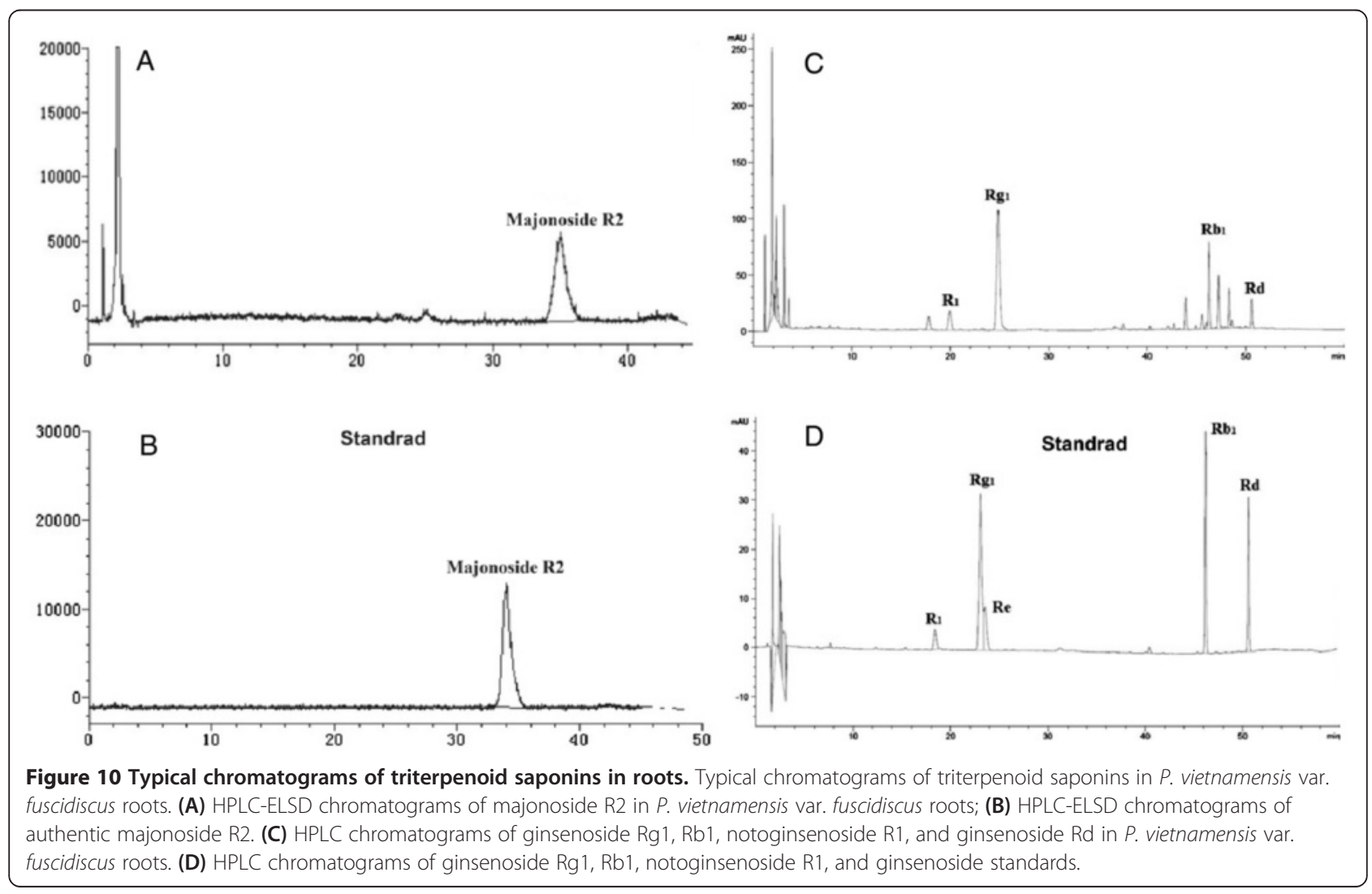




\section{Conclusions}

$P$. vietnamensis var. fuscidiscus exhibited remarkable disease resistance, and contains higher levels of ocotilloltype saponins. Thus, $P$. vietnamensis var. fuscidiscus is a suitable material for the study of ocotillol-type saponins biosynthesis and improvements of Panax plants. Because of the fact that $P$. vietnamensis var. fuscidiscus is a newly discovered variety of $P$. vietnamensis, no genomic information was available for this species. This is the first study performed on transcriptome sequencing of $P$. vietnamensis var. fuscidiscus using Illumina nextgeneration sequencing. In total, 126,758 unigenes were obtained. The large number of transcripts provided in this study not only facilitates the study of ocotillol-type saponins biosynthesis but also could provide opportunities to engineer microorganisms for the de novo production of active ingredients. Furthermore, numerous SSRs were identified and will be very useful for markerassisted selection breeding of this herb.

\section{Methods}

\section{Ethics statement}

No specific permits were required for the described field studies. No specific permissions were required for these locations and activities. The location is not privatelyowned or protected in any way and the field studies did not involve endangered or protected species.

\section{Plant material}

Four-year-old $P$. vietnamensis var. fuscidiscus plants were collected from Jinping County, Yunnan province, southwest of China (Latitude: $22^{\circ} 47^{\prime} 38^{\prime \prime} \mathrm{N}$, Longitude: $103^{\circ} 2^{\prime} 22^{\prime \prime}$ E, Altitude: $1690 \mathrm{~m}$ ), in May 2013. After morphological and molecular identification according the reference [24], the root tissues samples were collected separately from four randomly selected plant individuals. All samples were separately cut into small pieces, and parts of each sample were mixed with equivalent fresh weight ( $2 \mathrm{~g}$ ) for RNA isolation. The remaining materials were used for $S E$ gene cloning and RT-qPCR analysis. All samples were frozen immediately in liquid nitrogen and stored at $-80^{\circ} \mathrm{C}$ until use.

\section{RNA library construction and sequencing}

The total RNA was extracted from the mixed sample by using Trizol reagent (Invitrogen, Camarillo, CA, USA), following RNA purification by RNeasy MiniElute Cleanup Kit (Qiagen, Hilden, Germany), according to the manufacture's protocol. The RIN (RNA integrity number) values of the isolated RNA were determined by using Agilent 2100 Bioanalyzer (Santa Clara, CA, USA). Samples with RIN of more than 8 were used for further analysis. The construction of the libraries and the RNASeq were performed by CapitalBio Corporation (Beijing,
China). Firstly, poly (A) mRNA was purified from $20 \mu \mathrm{g}$ of total RNA using Oligo(dT) magnetic beads. Then, mRNA was fragmented into smaller pieces (200$700 \mathrm{bp}$ ), which were used for first-strand cDNA synthesis with reverse transcriptase and random hexamerprimer. The second-strand cDNA was synthesized using buffer, dNTPs, RNaseH and DNA polymerase. The short double-stranded cDNA fragments are purified with QiaQuick PCR extraction kit (Qiagen, Hilden, Germany) and resolved with EB buffer. These cDNA fragments underwent an end-repair process and poly(A) was added and then ligated with the Illumina paired-end sequencing adaptors. Subsequently, Ligation products were purified with magnetic beads and separated by agarose gel electrophoresis. A range of cDNA fragments $(200 \pm$ $25 \mathrm{bp}$ ) were excised from the gel and selected for PCR amplification as templates. The cDNA library was constructed with a fragment-length range of $200 \mathrm{bp}$ $( \pm 25 \mathrm{bp})$. Final, the cDNA library was sequenced on a paried-end flow cell using Illumina HiSeq ${ }^{\mathrm{mm}} 2000$ platform.

\section{Transcriptome data processing and assembly}

Before assembly, raw reads with adaptors and unknown nucleotides above $5 \%$ or those that were of low quality (containing more than $50 \%$ bases with Q-value $\leq 20$ ) were removed to obtain clean reads using a custom Perl script. Then the clean reads were de novo assembled using Trinity program [60] with default parameters. First, clean reads with a certain length of overlap were combined to form longer fragments without N, which were called contigs. These clean reads were then mapped back to corresponding contigs with paired-end reads to detect contigs from the same transcript as well as the distances between contigs, and their paired-end information was also used to fill gaps or to extend the sequences. Finally, these resultant sequences were clustered to remove redundant sequences using the TIGR gene Indices clustering tools (TGICL) [61] to form longer sequences without $\mathrm{N}$ and cannot be extended on either end. Such sequences are defined as unigenes.

\section{Functional annotation and prediction of CDS}

Functional annotations were performed by sequence comparison with public databases included the NCBI non-redundant nucleotide database (NT, by June 2012), non-redundant protein database (NR, by June 2012) (http://www.ncbi.nlm.nih.gov/), Swiss-Prot database (http://www.expasy.ch/sprot) and the Clusters of Orthologous Groups database (http://www.ncbi.nlm.nih. gov/COG/) [62] using BLASTN and BLASTX (http:// blast.ncbi.nlm.nih.gov/Blast.cgi), respectively, with an evalue of $1 \mathrm{e}^{-5}$. A Perl script was written to assign the functional class to unigenes. Unigenes were also 
compared with KEGG [63] using BLASTX at an evalues of less than $1 \mathrm{e}^{-10}$. A Perl script was used to retrieve KEGG Orthology (KO) information from blast result and then established pathway associations between unigenes and database. Based on the results of $\mathrm{Nr}$ database annotation, we use Blast2GO program [64] to perform GO annotation of unigenes. After acheiving GO annotation for every unigene, WEGO [65] software was used to perform GO classification and to draw GO tree. Moreover, the conserved domains/families of the assembled unigenes encoding proteins were searched against the Pfam database (version 26.0) [66] using Pfam_Scan script.

The coding sequence (CDS) for unigene was predicted by BlastX and ESTscan. The unigene sequences were searched against the Nr, COG, KEGG and Swiss-Prot protein databases using BLASTX (e-value $<10^{-5}$ ). Unigenes aligned to a higher priority database will not be aligned to lower priority database. The best alignment results were used to determine the sequence direction of unigenes. When a unigene could not be aligned to any database, ESTScan [67] program was used to predict coding regions and determine sequence direction.

\section{SSR detection and primer design}

Potential SSR markers were detected among the 126,758 unigenes using the MISA tool (http://pgrc.ipk-gatersleben.de/misa/). We searched for SSRs with motifs ranging from mono- to hexa-nucleotides in size. The minimum of repeat units were set as follows: ten repeat units for mono-nucleotide, six for di-nucleotides, and five for tri-, tetra-, penta- and hexa-nucelotides. Primer pairs were designed using Primer3 (http://bioinfo.ut.ee/ primer3-0.4.0/primer3/) with default parameters.

\section{Survey of SSR polymorphism}

A total of 30 primer pairs (Additional file 17) were randomly selected to evaluate their application and the polymorphism across $13 P$. vietnamensis var. fuscidiscus accessions (Additional file 18). Total DNA was isolated from $P$. vietnamensis var. fuscidiscus leaves using the CTAB method. PCR amplifications were conducted in a final volume of $20 \mu \mathrm{L}$ containing $1 \mu \mathrm{L} 2.5 \mathrm{mM}$ dNTPs, $1 \mu \mathrm{L}$ EasyTaq DNA polymerase (Beijing TransGen Biotech Co., Ltd. China), $2 \mu \mathrm{L} 10 \times$ EasyTaq buffer, $1 \mu \mathrm{L}$ of each primer $(10 \mu \mathrm{M}), 13 \mu \mathrm{L} \mathrm{ddH}_{2} \mathrm{O}$, and $1 \mu \mathrm{L}$ template DNA (approx. $10 \mathrm{ng} / \mu \mathrm{L}$ ). PCR was performed as follows: initial denaturation at $94^{\circ} \mathrm{C}$ for $2 \mathrm{~min}$, followed by 35 cycles of denaturation for $30 \mathrm{~s}$ at $94^{\circ} \mathrm{C}$, annealing for $30 \mathrm{~s}$ at different $\mathrm{Tm}$ depending on the gene, extension for $30 \mathrm{~s}$ at $72^{\circ} \mathrm{C}$, and a final step of elongation at $72^{\circ} \mathrm{C}$ for $5 \mathrm{~min}$. The separation of alleles was performed on $8 \%$ polyacrylamide gel. PCR products were mixed with an equal volume of loading buffer. The mixture was denatured at $95^{\circ} \mathrm{C}$ for $5 \mathrm{~min}$ before loading onto the gel.

\section{Data collection and analysis}

The presence of each single band was coded as 1 and its absence as 0 in a data matrix. Base on the binary data matrix, popgene program version 1.32 [68] was use to calculate genetic variation parameters, including observed number of alleles $(\mathrm{No})$, effective number of alleles $(\mathrm{Ne})$, Shannon's information index $(I)$, number of polymorphic loci $(N P)$ and percentage of polymorphic loci $(P P B)$. Allelic data were used to calculate the polymorphism Information Content (PIC) of each SSR marker by using the formula: $\mathrm{PIC}=1-\Sigma \mathrm{pi}^{2}$ ( $\mathrm{pi}$ is the frequency of $i^{\text {th }}$ allele for each locus) [69]. By NTSYS pc 2.1 program [70], Jaccard's genetic similarity coefficients were calculated and dendrogram of the $13 P$. vietnamensis var. fuscidiscus accessions was constructed by the UPGMA (un-weighted pair group method with arithmetic mean) clustering method.

\section{Full-length cDNA cloning of putative SE genes}

Total RNA was reverse transcribed to synthesize first strand cDNA using oligo dT primer and a PrimeScript ${ }^{\mathrm{TM}}$ II 1st Strand cDNA Synthesis Kit (TaKaRa, Dalian, China) according to the manufacturer's instructions. The RT-PCR products were used as template for cloning of PvfSE1, PvfSE2 and PvfSE3. The full-length cDNA sequences of PvfSE1, PvfSE2 and PvfSE3 were obtained from our transcriptome data. The specific primers (Additional file 19) used for the amplification of these genes were designed using primer3 program based on based on the predicted cDNA sequences and were then synthesized. PCRs were conducted in a total reaction volume of $25 \mu \mathrm{L}$, containing $1 \mu \mathrm{L}$ of cDNA, $0.5 \mu \mathrm{M}$ of each of the forward and reverse primers, $200 \mu \mathrm{M}$ of dNTPs, $5 \mu \mathrm{L}$ of $5 \times \mathrm{Q} 5$ Reaction Buffer, and $0.25 \mu \mathrm{L}$ of Q5 High-Fidelity DNA polymerase (NEB, Beijing, China). The PCR conditions are as follows: $94^{\circ} \mathrm{C}$ for $3 \mathrm{~min}$, followed by 35 cycles of $94^{\circ} \mathrm{C}$ for $1 \mathrm{~min}, 59^{\circ} \mathrm{C}$ for $1 \mathrm{~min}, 72^{\circ} \mathrm{C}$ for $5 \mathrm{~min}$, with a final 10 min extention at $72^{\circ} \mathrm{C}$. The PCR products were electrophoretically separatedon a $1 \%$ agarose gel, ligation into the pMD19-T vector (TaKaRa, Dalian, China) and were then subjected to automated DNA sequencing using the ABI 3730XL sequencer(Applied Biosystems, Foster City, USA).

\section{Phylogenetic analysis}

Phylogenetic analysis was performed based on the deduced amino acid sequences of Cytochrome P450 (CYP450) and UDP-glycosyltransferase (UGT) from $P$. vietnamensis var. fuscidiscus and other plants. All of the deduced amino acid sequences were aligned with Clustal 
$\mathrm{X}$ using the default parameters: gap opening penalty, 10; gap extension penalty, 0.1 ; and delay divergent cutoff, $25 \%$, and evolutionary distances were computed using MEGA5.10 with the Poisson correction method. For the phylogenetic analysis, a neighbor-joining tree was constructed using MEGA5.0. Bootstrap values obtained after 1000 replications are indicated on the branches. The scale repesents 0.1 amino acid substitutions per site.

\section{RT-qPCR analysis}

Ten unigenes with potential roles in ginsenoside biosynthesis were chosen for validation using RT-qPCR with gene specific primers designed with Primer3 software. All the primers sequences used for the RT-qPCR analysis are shown in Additional file 20. Total RNA from different organs (roots, hairy roots, stems and leaves) of $P$. vietnamensis var. fuscidiscus were extracted individually using Trizol Kit (Promega, USA) following the manufacturer's protocol. Subsequently, RNA was treated with $4 \times$ gDNA wiperMix at $42^{\circ} \mathrm{C}$ for $2 \mathrm{~min}$ to remove DNA. The purified RNA (1ug) was reverse transcribed to cDNA using HiScript QRT SuperMix for qPCR (Vazyme, Nanjing, China). The qPCR reactions were performed in a $20 \mu \mathrm{l}$ volume composed of $2 \mu \mathrm{l}$ of cDNA, $0.4 \mu \mathrm{l}$ of each primer, and $10 \mu \mathrm{l} 2 \times$ SYBR Green Master mix (TaKaRa) in Roche LightCycler 2.0 system (Roche Applied Science, Branford, CT). PCR amplification was performed under the following conditions: $30 \mathrm{~s}$ at $94^{\circ} \mathrm{C}$, followed by 45 cycles of $94^{\circ} \mathrm{C}$ for $20 \mathrm{~s}, 55^{\circ} \mathrm{C}$ for $20 \mathrm{~s}$, and $72^{\circ} \mathrm{C}$ for $30 \mathrm{~s}$. Three technical replications were performed for all quantitative PCRs. The phosphomevalonate kinase (PMK) gene, which was found in our transcriptome database, was chosen as reference gene control for normalization after the expressions of three reference genes (actin, GAPDH, and PMK) were compared in different tissues. The relative changes in gene expression levels were calculated using the $2^{-\Delta \Delta \mathrm{Ct}}$ method.

\section{HPLC- ELSD analysis of majonoside R2}

The dried powder of $P$. vietnamensis var. fuscidiscus roots $(0.11 \mathrm{~g})$ were extracted by sonication with $50 \mathrm{ml}$ of methanol for $45 \mathrm{~min}$, let cool, then weighed and the weight of methanol to complement the weight loss, shake, with $0.45 \mu \mathrm{m}$ microporous membrane filtration, and $10 \mu \mathrm{L}$ of filtrate was analyzed by HPLC-ELSD. For majonoside R2 determination, a Shimadzu LC 20A HPLC system (Shimadzu, Kyoto, Japan) with a Sedex 75 evaporative light scattering detector (Sedere, Alfortville, France) was used. Chromatographic separation was performed on an Waters symmetry shield ${ }^{\mathrm{m}} \mathrm{RP}_{10}(4.6 \mathrm{~mm} \times$ $250 \mathrm{~mm}, 5.0 \mathrm{um}$, Milford, MA, USA) column maintained at $30^{\circ} \mathrm{C}$. The mobile phase was acetonitrile-water $(19.5: 80.5, \mathrm{v} / \mathrm{v})$, and the flow rate was $1 \mu \mathrm{L} / \mathrm{min}$. The drift tube temperature of ELSD was set at $40^{\circ} \mathrm{C}$ and nebulizer nitrogen gas flow-rate was $1.5 \mathrm{l} / \mathrm{min}$ and gain of 9 . Authentic majonoside R2 was provided by Yunnan Institute for Food and Drug Control (Kunming, Yunnan, China).

\section{HPLC analysis of other four triterpene saponins}

In brief, the dried powder of $P$. vietnamensis var. fuscidiscus roots $(0.6 \mathrm{~g})$ were extracted with $40 \mathrm{~mL}$ of $100 \%$ $\mathrm{MeOH}$ for $30 \mathrm{~min}$ and sonicated for $60 \mathrm{~min}$ and then diluted to $50 \mathrm{~mL}$ with $\mathrm{MeOH}$. The methanol extract was filtered through a $0.45 \mu \mathrm{m}$ membrane filter and $10 \mu \mathrm{L}$ of filtrate was directly injected into the HPLC system.

Quantitative analysis of the remaining four triterpene saponins (ginsenoside Rg1, Rb1, notoginsenoside R1, and ginsenoside $\mathrm{Rd}$ ) in the roots of this herb was performed on Agilent 1260 HPLC systems (Agilent Technologies, Santa Clara, CA, USA). The chromatographic column Agilent Zorbar SB-C 18 (250 mm $\times$ $4.6 \mathrm{~mm}, 5 \mu \mathrm{m}$, Agilent Technologies, Santa Clara, CA, USA) was used and the column temperature was maintained at $30{ }^{\circ} \mathrm{C}$. The flow rate was fixed at $1 \mathrm{~mL} / \mathrm{min}$, and the mobile phase consisted of acetonitrile (A) and water (B) and separation was achieved using the following gradient system: $85 \% \mathrm{~B}$ at $0 \mathrm{~min}, 80 \% \mathrm{~B}$ at $5 \mathrm{~min}$, $77 \% \mathrm{~B}$ at $30 \mathrm{~min}, 60 \% \mathrm{~B}$ at $50 \mathrm{~min}$, and $60 \% \mathrm{~B}$ at $55 \mathrm{~min}$. Detection was performed at $203 \mathrm{~nm}$ for the remaining four triterpene saponins. Authentic ginsenoside Rg1, Rb1, notoginsenoside R1, and ginsenoside Rd were purchased from J\&K Scientific Ltd (Beijing, PR China).

\section{Additional files}

Additional file 1: Assessment of assembly quality.

Additional file 2: Comparison of $\mathrm{P}$. vietnamensis var. fuscidiscus unigenes to orthologous $P$. notoginseng coding sequences.

Additional file 3: Venn diagram results from diverse databases.

Additional file 4: Comparison of unigene length between hit and no hit unigenes.

Additional file 5: Characterization of searching the assembled unigenes against NCBI Nr and Swiss-Prot protein databases.

Additional file 6: Top-hit species distribution for sequences from $P$. vienamensis var. fuscidiscus submitted BLASTX against the NCBI-Nr database.

Additional file 7: Gene Ontology classification.

Additional file 8: List of Pfam domain families assigned to $P$. vietnamensis var. fuscidiscus unigenes.

Additional file 9: Top 20 Pfam domainsfamilies predcted in P. vienamensis var. fuscidiscus.

Additional file 10: Mapping of P.vietnamensis var. fuscidiscus unique sequences to KEGG biochemical pathways.

Additional file 11: Information of SSR derived from all unigene.

Additional file 12: Sequence informations of SSR primers. 


\section{Additional file 13: Characteristics of 15 polymorphic EST-SSR primer pairs in $13 P$. vietnamensis var. fuscidiscus accessions.}

Additional file 14: The main identified triterpene saponin biosynthetic genes from $P$. vietnamensis var. fuscidiscus unigenes.

Additional file 15: Cytochrome P450 discovery.

Additional file 16: The main identified glycosyltransferase genes from $P$. vietnamensis var. fuscidicus unigenes.

Additional file 17: SSR primer pairs validated in this study. Additional file 18: $P$. vietnamensis var. fuscidiscus germplasms for polymorphism validation with EST-SSRs.

Additional file 19: Gene-specific used for reverse-transcriptase PCR assays.

Additional file 20: Primers used for gene expression analysis by reverse transcription quantitative real-time PCR (RT-qPCR).

\section{Abbreviations}

NCBI: National Center for Biotechnology Information; Nr: Non-redundant protein; COG: Cluster of Orthologous Groups; GO: Gene Ontology; KEGG: Kyoto Encyclopedia of Genes and Genomes; SSRs: Simple sequence repeats; CDNA: Complementary DNA; BLAST: Basic Local Alignment Search Tool; CDS: Coding sequence; bp: Base pair; AACT: Acetyl-CoA acetyltransferase; $\beta$-AS: $\beta$-amyrin synthase; DMAPP: Dimethylallyl diphosphate; DS: Dammarenediol-II synthase; FPP: Farnesyl diphosphate FPPS: Farnesyl diphosphate synthase; Glc: Glucose; GPP: Geranyl pyrophosphate; GGPP: Geranylgeranyl diphosphate; GGPPS: Geranylgeranyl pyrophosphate synthase; GT: Glycosyltransferase; HMG-CoA: 3-hydroxy-3methylglutaryl coenzyme A; HMGR: HMG-CoA reductase; HMGS: HMG-CoA synthase; IPP: Isopentenyl diphosphate; IPPI: IPP isomerase; MVD: Mevalonate diphosphate decarboxylase; MVK: Mevalonate kinase; P450: Cytochrome P450; PMK: Phosphomevalonate kinase; SE: Squalene epoxidase; SS: Squalene synthase; RT-PCR: Reverse transcription PCR; RT-qPCR: Reverse transcription quantitative real-time PCR; HPLC-ELSD: High performance liquid chromatography with evaporative light scattering detector; HPLC: High performance liquid chromatography.

\section{Competing interests}

The authors declare that they have no competing interests.

\section{Authors' contributions}

This study was conceived by JWC and SCY. The plant material preparation were carried out by CHM, QYT, MHH and XZX, GHZ, JJZ and NHJ analysed the RNA-Seq data. GHZ and NHJ drafted the manuscript. JWC revised the manuscript. All authors read and approved the final manuscript.

\section{Acknowledgements}

This work was funded by the National "Twelfth Five-Year" Support Project of China (Grant No. 2011BAl13B01), the Important Specific Projects of the Development and Reform Commission of Yunnan Province (Grant No. 20112513), the National Natural Science Foundation of China (Grant No. 81303164 and 81360609), the project of young and middle-aged talent of Yunnan province (Grant No. 2014HB011), and the Joint Funds of the National Natural Science Foundation of China (Grant No. U1402262).

Received: 18 August 2014 Accepted: 9 February 2015 Published online: 08 March 2015

\section{References}

1. Christensen LP. Ginsenosides chemistry, biosynthesis, analysis, and potential health effects. Adv Food Nutr Res. 2009;55:1-99.

2. Zhu S, Zou K, Fushimi H, Cai S, Komatsu K. Comparative study on triterpene saponins of ginseng drugs. Planta Med. 2004;70(7):666-77.

3. Wang CZ, McEntee E, Wicks S, Wu JA, Yuan CS. Phytochemical and analytical studies of Panax notoginseng (Burk.) F. H. Chen. J Nat Med. 2006:60:97-106.

4. Konoshima T, Takasaki M, Tokuda H, Nishino H, Duc NM, Kasai R, et al. Anti- tumor- promoting activity of majonoside- $R_{2}$ from Vietnamese ginseng, Panax vietnamensis Ha et Grushv. (I). Biol Pharm Bull. 1998;21(8):834-8.
5. Konoshima T, Takasaki M, Ichiishi E, Murakami T, Tokuda H, Nishino H, et al. Cancer chemopreventive activity of majonoside- $\mathrm{R}_{2}$ from Vietnamese ginseng, Panax vietnamensis. Cancer Lett. 1999;147(1-2):11-6.

6. Tran QL, Adnyana IK, Tezuka Y, Nagaoka T, Tran QK, Kadota S. Triterpene saponins from vietnamese ginseng (Panax vietnamensis) and their hepatocytoprotective activity. J Nat Prod. 2001;64(4):456-61

7. Haralampidis K, Trojanowska M, Osbourn AE. Biosynthesis of triterpenoid saponins in plants. Adv Biochem Eng/Biotechnol. 2002;75:31-49.

8. Sawai S, Saito K. Triterpenoid biosynthesis and engineering in plants. Front Plant Sci. 2011;2(25):1-8.

9. Jung JD, Park HW, Hahn Y, Hur CG, In DS, Chung HJ, et al. Discovery of genes for ginsenoside biosynthesis by analysis of ginseng expressed sequence tags. Plant Cell Rep. 2003;22(3):224-30.

10. Lee $\mathrm{MH}$, Jeong JH, Seo JW, Shin CG, Kim YS, In JG, et al. Enhanced triterpene and phytosterol biosynthesis in Panax ginseng overexpressing squalene synthase gene. Plant Cell Physiol. 2004;45(8):976-84.

11. Choi DW, Jung J, Ha Yl, Park HW, In DS, Chung HJ, et al. Analysis of transcripts in methyl jasmonate-treated ginseng hairy roots to identify genes involved in the biosynthesis of ginsenosides and other secondary metabolites. Plant Cell Rep. 2005;23(8):557-66.

12. Han JY, Kwon YS, Yang DC, Jung YR, Choi YE. Expression and RNA interference- induced silencing of the dammarenediol synthase gene in Panax ginseng. Plant Cell Physiol. 2006:47:1653-62.

13. Han JY, Kim HJ, Kwon YS, Choi YE. The Cyt P450 enzyme CYP716A47 catalyzes the formation of protopanaxadiol from dammarenediol--l during ginsenoside biosynthesis in Panax ginseng. Plant Cell Physiol. 2011;52 (12):2062-73.

14. Han JY, Hwang HS, Choi SW, Kim HJ, Choi YE. Cytochrome P450 CYP716A53V2 catalyzes the formation of protopanaxatriol from protopanaxadiol during ginsenoside biosynthesis in Panax ginseng. Plant Cell Physiol. 2012;53(9):1535-45.

15. Han JY, Kim MJ, Ban YW, Hwang HS, Choi YE. The involvement of $\beta$-amyrin 28-oxidase (CYP716A52V2) in oleanane-type ginsenoside biosynthesis in Panax ginseng. Plant Cell Physiol. 2013;54(12):2034-46.

16. Tansakul P, Shibuya M, Kushiro T, Ebizuka Y. Dammarenediol-Il synthase, the first dedicated enzyme for ginsenoside biosynthesis, in Panax ginseng. FEBS Lett. 2006;580(22):5143-9.

17. Hu FX, Zhong JJ. Jasmonic acid mediates gene transcription of ginsenoside biosynthesis in cell cultures of Panax notoginseng treated with chemically synthesized 2-hydroxyethyl jasmonate. Process Biochem. 2008;43(1):113-8.

18. Sun C, Li Y, Wu Q, Luo H, Sun Y, Song J, et al. De novo sequencing and analysis of the American ginseng root transcriptome using a GS FLX Titanium platform to discover putative genes involved in ginsenoside biosynthesis. BMC Genomics. 2010;11:262.

19. Wu Q, Song J, Sun Y, Suo F, Li C, Luo H, et al. Transcript profiles of Panax quinquefolius from flower, leaf and root bring new insights into genes related to ginsenosides biosynthesis and transcriptional regulation. Plant Physiol. 2010;138(2):134-49.

20. Chen S, Luo H, Li Y, Sun Y, Wu Q, Niu Y, et al. 454 EST analysis detects genes putatively involved in ginsenoside biosynthesis in Panax ginseng. Plant Cell Rep. 2011;30(9):1593-601.

21. Luo H, Sun C, Sun Y, Wu Q, Li Y, Song J, et al. Analysis of the transcriptome of Panax notoginseng root uncovers putative triterpene saponin-biosynthetic genes and genetic markers. BMC Genomics. 2011;12:S5.

22. Li C, Zhu Y, Guo X, Sun C, Luo H, Song J, et al. Transcriptome analysis reveals ginsenosides biosynthetic genes, microRNAs and simple sequence repeats in Panax ginseng C.A. Meyer. BMC Genomics. 2013;14:245.

23. Shan H, Segura MJ, Wilson WK, Lodeiro S, Matsuda SP. Enzymatic cyclization of dioxidosqualene to heterocyclic triterpenes. J Am Chem Soc. 2005;127 (51):18008-9.

24. Zhu S, Fushimi H, Cai S, Chen H, Komatsu K. A new variety of the genus Panax from southern Yunnan, China and its nucleotide sequences of $18 \mathrm{~S}$ ribosomal RNA gene and matK gene. Journal of Japanese Botany. 2003;78(2):86-94.

25. Li R, Li Y, Kristiansen K, Wang J. SOAP: short oligonucleotide alignment program. Bioinformation. 2008;24:713-4.

26. Hao D, Ge G, Xiao P, Zhang Y, Yang $L$. The first insight into the tissue specific taxus transcriptome via illumina second generation sequencing. PLoS One. 2011;6:e21220.

27. Shu S, Chen B, Zhou M, Zhao X, Xia H. De novo sequencing and transcriptome analysis of Wolfiporia cocosto reveal genes related to biosynthesis of triterpenoids. PLoS One. 2013;8:e71350. 
28. Huang L, Yang $X$, Sun P, Tong W, Hu S. The first illumina-based de novo transcriptome sequencing and analysis of safflower flowers. PLoS One. 2012;7:e38653

29. Shikanai T, Okuda K. In vitro rna-binding assay for studying trans-factors for RNA editing in chloroplasts. Methods Mol Biol. 2011;774:199-208.

30. Zehrmann A, Verbitskiy D, Hartel B, Brennicke A, Takenaka M. PPR proteins network as site-specific RNA editing factors in plant organelles. RNA Biol. 2011:8:67-70.

31. Takenaka M, Verbitskiy D, Zehrmann A, Brennicke A. Reverse genetic screening identifies five E-class ppr proteins involved in RNA editing in mitochondria of Arabidopsis thaliana. J Biol Chem. 2010;285:27122-9.

32. Fujii S, Small I. The evolution of RNA editing and pentatricopeptide repeat genes. New Phytol. 2011;191:37-47.

33. Kobe B, Kajava AV. The leucine-rich repeat as a protein recognition motif. Curr Opin Struct Biol. 2001;11:725-32.

34. Neer EJ, Schmidt CJ, Nambudripad R, Smith TF. The ancient regulatory-protein family of WD-repeat proteins. Nature. 1994;371:297-300.

35. Maier D, Nagel AC, Gloc H, Hausser A, Kugler SJ. Protein kinase D regulates several aspects of development in Drosophila melanogaster. BMC Dev Biol. 2007;7:74.

36. Ahier A, Rondard P, Gouignard N, Khayath N. A new family of receptor tyrosine kinases with a Venus Flytrap binding domain in insects and other invertebrates activated by amino acids. PLoS One. 2009;4:e5651.

37. Corey EJ, Matsuda SP, Bartel B. Isolation of an Arabidopsis thaliana gene encoding cycloartenol synthase by functional expression in a yeast mutant lacking lanosterol synthase by the use of a chromatographic screen. Proc Natl Acad Sci U S A. 1993;90(24):11628-32.

38. Kushiro T, Shibuya M, Ebizuka Y. $\beta$-amyrin synthase-cloning of oxidosqualene cyclase that catalyzes the formation of the most popular triterpene among higher plants. Eur J Biochem. 1998;256(1):238-44

39. Herrera JB, Bartel B, Wilson WK, Matsuda SP. Cloning and characterization of the Arabidopsis thaliana lupeol synthase gene. Phytochemistry. 1998;49 (7):1905-11.

40. Augustin JM, Kuzina V, Andersen SB, Bak S. Molecular activities, biosynthesis and evolution of triterpenoid saponins. Phytochemistry. 2011;72(6):435-57.

41. Carelli M, Biazzi E, Panara F, Tava A, Scaramelli L, Porceddu A, et al. Medicago truncatula CYP716A12 is a multifunctional oxidase involved in the biosynthesis of hemolytic saponins. Plant Cell. 2011:8:3070-81.

42. Fukushima EO, Seki H, Ohyama K, Ono E, Umemoto N, Mizutani M, et al. CYP716A subfamily members are multifunctional oxidases in triterpenoid biosynthesis. Plant Cell Physiol. 2011;12:2050-61.

43. Seki H, Ohyama K, Sawai S, Mizutani M, Ohnishi T, Sudo H, et al. Licorice $\beta$-amyrin 11-oxidase, a cytochrome P450 with a key role in the biosynthesis of the triterpene sweetener glycyrrhizin. Proc Natl Acad Sci. 2008;105(37):14204-9.

44. Seki H, Sawai S, Ohyama K, Mizutani M, Ohnishi T, Sudo H, et al. Triterpene functional genomics in licorice for identification of CYP72A154 involved in the biosynthesis of glycyrrhizin. Plant Cell. 2011;11:4112-23.

45. Shibuya M, Hoshino M, Katsube $Y$, Hayashi H, Kushiro T, Ebizuka $Y$. Identification of $\beta$-amyrin and sophoradiol 24-hydroxylase by expressed sequence tag mining and functional expression assay. FEBS J. 2006;273 (5):948-59.

46. Fukushima EO, Seki H, Sawai S, Suzuki M, Ohyama K, Saito K, et al. Combinatorial biosynthesis of legume natural and rare triterpenoids in engineered yeast. Plant Cell Physiol. 2013;54(5):740-9.

47. Qi X, Bakht S, Qin B, Leggett M, Hemmings A, Mellon F, et al. A different function for a member of an ancient and highly conserved cytochrome P450 family: from essential sterols to plant defense. Proc Natl Acad Sci U S A. 2006;103(49):18848-53.

48. Kunii M, Kitahama Y, Fukushima EO, Seki H, Muranaka T, Yoshida Y, et al. $\beta$-Amyrin oxidation by oat CYP51H10 expressed heterologously in yeast cells: the first example of CYP51-dependent metabolism other than the 14-demethylation of sterol precursors. Biol Pharm Bull. 2012;35(5):801-4.

49. Geisler K, Hughes RK, Sainsbury F, Lomonossoff GP, Rejzek M, Fairhurst S, et al. Biochemical analysis of a multifunctional cytochrome P450 (CYP51) enzyme required for synthesis of antimicrobial triterpenes in plants. Proc Natl Acad Sci U S A. 2013;110(35):E3360-7.

50. Field B, Fiston-Lavier AS, Kemen A, Geisler K, Quesneville H, Osbourn AE. Formation of plant metabolic gene clusters within dynamic chromosomal regions. Proc Natl Acad Sci U S A. 2011;108(38):16116-21.
51. Li L, Cheng H, Gai J, Yu D. Genome-wide identification and characterization of putative cytochrome P450 genes in the model legume Medicago truncatula. Planta. 2007;226(1):109-23.

52. Xiang L, Guo X, Niu YY, Chen SL, Luo HM. Full-length cDNA cloning and bioinformatics analysis of PnUGT1 gene in Panax notoginseng. Acta Pharmaceutica Sinica. 2012;47(8):1085-91.

53. Achnine L, Huhman DV, Farag MA, Sumner LW, Blount JW, Dixon RA. Genomics-based selection and functional characterization of triterpene glycosyltransferases from the model legume Medicago truncatula. Plant J. 2005;41(6):875-87.

54. Augustin JM, Drok S, Shinoda T, Sanmiya K, Nielsen JK, Khakimov B, et al. UDP-glycosyltransferases from the UGT73C subfamily in Barbarea vulgaris catalyze sapogenin 3-O-glucosylation in saponin-mediated insect resistance. Plant Physiol. 2012;160(4):1881-95.

55. Kohara A, Nakajima C, Hashimoto K, Ikenaga T, Tanaka H, Shoyama Y, et al. A novel glucosyltransferase involved in steroid saponin biosynthesis in Solanum aculeatissimum. Plant Mol Biol. 2005;57(2):225-39.

56. Naoumkina MA, Modolo LV, Huhman DV, Urbanczyk-Wochniak E, Tang Y, Sumner LW, et al. Genomic and coexpression analyses predict multiple genes involved in triterpene saponin biosynthesis in Medicago truncatula. Plant Cell. 2010:22(3):850-66.

57. Sayama T, Ono E, Takagi K, Takada Y, Horikawa M, Nakamoto Y, et al. The Sg-1 glycosyltransferase locus regulates structural diversity of triterpenoid saponins of soybean. Plant Cell. 2012;24(5):2123-38.

58. Meesapyodsuk D, Balsevich J, Reed DW, Covello PS. Saponin biosynthesis in Saponaria vaccaria. cDNAs encoding $\beta$-amyrin synthase and a triterpene carboxylic acid glucosyltransferase. Plant Physiol. 2007;143(2):959-69.

59. Kim TD, Han JY, Huh GH, Choi YE. Expression and functional characterization of three squalene synthase genes associated with saponin biosynthesis in Panax ginseng. Plant Cell Physiol. 2011;52(1):125-37.

60. Grabherr MG, Haas BJ, Yassour M, Levin JZ, Thompson DA. Full-length transcriptome assembly from RNA-Seqdata without a reference genome. Nature Biotechnology. 2011;29(7):644-52.

61. Pertea G, Huang X, Liang F, Antonescu V, Sultana R. TIGR gene indices clustering tools (TGICL):a software system for fast clustering of large EST datasets. Bioinformatics. 2003;19:651-2.

62. Tatusov RL, Koonin EV, Lipman DJ. A genomic perspective on protein families. Science. 1997;278(5338):631-7.

63. Kanehisa M, Goto S, Hattori M, Aoki-Kinoshita KF, Itoh M. From genomics to chemical genomics: new developments in KEGG. Nucleic Acids Res. 2006;34: D354-7.

64. Conesa A, Gotz S, Garcia-Gomez JM, Terol J, Talon M. Blast2GO: a universal tool for annotation, visualization and analysis in functional genomics research. Bioinformatics. 2005:21:3674-6.

65. Ye J, Fang L, Zheng H, Zhang Y, Chen J. WEGO: a web tool for plotting GO annotations. Nucleic Acids Res. 2006:34:293-7.

66. Finn RD, Bateman A, Clements J, Coggill P, Eberhardt RY. Pfam: the protein families database. Nucleic Acids Res. 2014:42:D222-30.

67. Iseli C, Jongeneel CV, Bucher P. ESTScan: a program for detecting, evaluating, and reconstructing potential coding regions in EST sequences. Proc Int Conf Intell Syst Mol Biol. 1999;1:138-48.

68. Yeh FC, Boyle TJB. Population genetic analysis of co-dominant and dominant markers and quantitative traits. Belg J Bot. 1997;129:157.

69. Weir BS. Genetic data analysis: methods for discrete population genetic data. Sunderland, Massachusetts: Sinauer Associates, Inc. Publishers; 1990.

70. Rohlf FJ. NTSYSpc, numerical taxonomy and multivariate analysis system, version 2.1. New York: Exeter Software Inc: 2000 